Copyright

by

Pranav Madhav Karve

2009 
The Thesis Committee for Pranav Madhav Karve certifies that this is the approved version of the following thesis:

The inverse medium problem for Timoshenko beams and frames: damage detection and profile reconstruction in the time-domain

APPROVED BY

SUPERVISING COMMITTEE:

Loukas F Kallivokas, Supervisor

Lance Manuel 
The inverse medium problem for Timoshenko beams and frames: damage detection and profile reconstruction in the time-domain

\author{
by \\ Pranav Madhav Karve, B.E.
}

\author{
THESIS \\ Presented to the Faculty of the Graduate School of \\ The University of Texas at Austin \\ in Partial Fulfillment \\ of the Requirements \\ for the Degree of \\ MASTER OF SCIENCE IN ENGINEERING
}

THE UNIVERSITY OF TEXAS AT AUSTIN

December 2009 
Dedicated to Aai-Baba and Shraddha. 


\section{Acknowledgments}

First and foremost, I wish to thank Dr. Kallivokas for giving me the opportunity to work with him. He was always available to answer my questions regarding this research or otherwise. This thesis would not have been possible without his patience and invaluable guidance. I am grateful to Dr. Manuel for his observations and comments as the second reader of this thesis.

I would like to thank Dr. Seong-Won Na for his initial assistance in this work and my lab mates - Jun Won Kang, Chanseok Jeong, and Sezgin Kucukcoban for the numerous discussions instrumental in my understanding of inverse problems. I am fortunate to have many friends who have helped me, either directly or indirectly, in this work and I wish to thank all of them.

Lastly, I am obliged to my family for their unyielding support and love. 


\title{
The inverse medium problem for Timoshenko beams and frames: damage detection and profile reconstruction in the time-domain
}

\author{
Pranav Madhav Karve, M.S.E. \\ The University of Texas at Austin, 2009 \\ Supervisor: Loukas F Kallivokas
}

We discuss a systematic methodology that leads to the reconstruction of the material profile of either single, or assemblies of one-dimensional flexural components endowed with Timoshenko-theory assumptions. The probed structures are subjected to user-specified transient excitations: we use the complete waveforms, recorded directly in the time-domain at only a few measurement stations, to drive the profile reconstruction using a partial-differentialequation-constrained optimization approach. We discuss the solution of the ensuing state, adjoint, and control problems, and the alleviation of profile multiplicity by means of either Tikhonov or Total Variation regularization. We report on numerical experiments using synthetic data that show satisfactory reconstruction of a variety of profiles, including smoothly and sharply varying profiles, as well as profiles exhibiting localized discontinuities. The method is well suited for imaging structures for condition assessment purposes, and 
can handle either diffusive or localized damage without need for a reference undamaged state. 


\section{Table of Contents}

Acknowledgments $\quad$ v

Abstract vi vi

List of Tables $\quad$ X

List of Figures $\quad$ xi

Chapter 1. Introduction 1

$\begin{array}{lll}\text { Chapter 2. The forward problem } & 9\end{array}$

$\begin{array}{lll}\text { Chapter 3. The inverse problem } & 12\end{array}$

3.1 Objective functional . . . . . . . . . . . . . . . . . . . 12

3.1.1 Regularization . . . . . . . . . . . . . . . 13

3.2 PDE-constrained optimization . . . . . . . . . . . . . . . 14

3.2.1 Lagrangian functional . . . . . . . . . . . . . . . . . 14

3.2.2 The first optimality condition . . . . . . . . . . . 15

3.2.3 The second optimality condition . . . . . . . . . . 16

3.2.4 The third optimality condition: . . . . . . . . . . 25

$\begin{array}{lll}\text { Chapter } 4 . & \text { The inversion process } & 29\end{array}$

4.1 The state problem - semi-discrete form . . . . . . . . . . . 29

4.2 The adjoint problem - semi-discrete form . . . . . . . . . . 33

4.3 State and adjoint time integration . . . . . . . . . . . . 35

4.3.1 Time integration for the state problem . . . . . . . 35

4.3.2 Time integration for the adjoint problem . . . . . . . 36

4.4 Inversion parameter updates . . . . . . . . . . . . . 37

4.4.1 Gradient-based scheme for inversion variable updates . . 39 
4.4.2 Weak-form-based scheme for inversion variable updates 42

4.5 Regularization factor continuation . . . . . . . . . . . . . . 44

$\begin{array}{lll}\text { Chapter 5. Numerical results } & 46\end{array}$

$\begin{array}{ll}\text { Chapter 6. Extensions } & 57\end{array}$

6.1 Material profile reconstruction for frames . . . . . . . . . 57

6.2 Inversion variables other than Young's modulus . . . . . . . 66

$\begin{array}{lll}\text { Chapter 7. Conclusions } & 67\end{array}$

$\begin{array}{lr}\text { Bibliography } & 69\end{array}$

$\begin{array}{ll}\text { Vita } & 73\end{array}$ 


\section{List of Tables}

2.1 Boundary conditions for Timoshenko beam (Huang [7]) . . . . 11

4.1 Inversion algorithm (Kang and Kallivokas [8]) . . . . . . . . 41

5.1 Example characteristics: smallest wavelength $\lambda$, element size $h_{e}$, time-step $\Delta t$, total observation time $T$, number of iterations 48

6.1 Sensor locations for the frame shown in Fig. $6.5 \ldots 60$ 


\section{List of Figures}

2.1 Schematic of Timoshenko beam: initially plane cross-section AA' remains plane post-deformation with shear effects taken into account . . . . . . . . . . . . . . . .

4.1 Nodes associated with the element interpolants for the nodal deflections $w$, nodal slopes $\psi$, and nodal Young's moduli $E$. .

5.1 Reconstruction of a smooth target profile for a simply-supported beam: excitation, uniform load, sensor distribution, TN regularization . . . . . . . . . . . . . .

5.2 Reconstruction of a smooth target profile for a simply-supported beam: excitation, point load location, sensor distribution, TN regularization . . . . . . . . . . . . . .

5.3 Reconstruction of a sharp target profile for a simply-supported beam: excitation, uniform load, sensor distribution, TV regu-

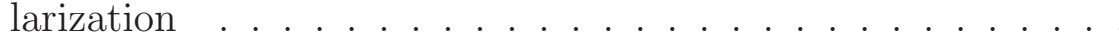

5.4 Reconstruction of a sharp target profile for a simply-supported beam: excitation, point load location, sensor distribution, TV regularization . . . . . . . . . . . . . .

5.5 Reconstruction of a multiply-sharp target profile for a simplysupported beam: excitation, uniform load, sensor distribution, TV regularization . . . . . . . . . . . . . .

5.6 Reconstruction of localized damage for a simply-supported beam: excitation, point load location, sensor distribution, TV regularization . . . . . . . . . . . . . . . . .

5.7 Reconstruction of a sharp target profile for a simply-supported beam with $10 \%$ Gaussian noise in the data: excitation, point load location, sensor distribution, TV regularization . . . . . .

6.1 Prototype portal frame . . . . . . . . . . . . . . 57

6.2 Reconstruction of a smooth target profile for a prototype portal frame: excitation, point load location, sensor distribution, TN regularization . . . . . . . . . . . . . . 
6.3 Reconstruction of a sharp target profile for a prototype portal frame: excitation, point load location, sensor distribution, TV regularization . . . . . . . . . . . . .

6.4 Reconstruction of localized damage for a prototype portal frame: excitation, point load location, sensor distribution, TV regularization . . . . . . . . . . . . . . . . .

6.5 Time signal, geometry, load and sensor locations for material profile reconstruction of a frame . . . . . . . . . . . .

6.6 Reconstruction of material profile including localized damage for the frame in Fig. 6.5 using TV regularization . . . . . . . . 


\section{Chapter 1}

\section{Introduction}

Non-destructive testing of structural components is at the very core of structural health monitoring, system identification, and diagnostics for infrastructure systems. The goal of condition assessment is the localization and quantification of alterations imparted on a (structural) system between the time it was placed in operation (or even its original design state), and the moment condition assessment is requested. To date, a plethora of condition assessment methods have been used, where methods relying on the analysis of the dynamics of a structure, following its probing by a time-dependent excitation, continue to be dominant. Probing refers to targeting the structure using acoustic, electromagnetic, or stress waves, and/or their combination. The analysis of the resulting dynamics refers to post-processing the structure's response, aiming at reconstructing the profile; typically, the response has been collected at a few points, a limited area, or in general, a subset of the structure's bounding surface. One could broadly classify these dynamicsbased methods into methods relying on the analysis of the structure's modal parameters (eigenfrequencies, mode shapes, curvatures of mode shapes, etc), or on the analysis of travel times associated with the induced wave motion. By and large, both classes of methodologies underutilize either the frequency 
spectrum, or the transient record: analyses based on modal parameters rely typically on a few modes, irrespective of the particular modal quantity used to drive the detection process, whereas analyses based on the propagation of waves use travel times and first arrivals to infer the location of structural flaws (e.g. cracks).

Of particular interest in this article is the exploitation of the complete waveforms for damage detection, or more broadly, for reconstructing the material profile of a probed structure consisting of Timoshenko-type beams. The approach formally gives rise to an inverse problem, which, even though it lacks the deterministic character of the aforementioned methodologies, it takes advantage of the complete waveforms and uses the totality of the recorded information to infer the profile. In this sense, there is reasonable expectation for a more accurate profile reconstruction, when compared to other approaches, at the (affordable) expense of algorithmic complexity. The battleground for full waveform methods has been the geophysical exploration arena, where the goal is to recover the material distribution by wave probing, for the purpose of identifying hydrocarbon pockets embedded in highly heterogeneous domains. From a methodological point of view, structural systems are similar to geologic formations, under the unifying assumption of a linear elastic behavior during wave probing.

Next, we highlight developments related to dynamics-based probing, to place the proposed methodology in context. Early efforts were primarily focused on damage detection given experimentally determined modal param- 
eters. Pioneering work on this class of problems involved the use of eigenfrequencies. Adams et. al. [1] devised a damage detection scheme based on shifts in the natural frequencies between the virgin and damaged state of a structure, for which a priori knowledge of the virgin state was necessary (in fact, the virgin state was assumed to correspond to the theoretically perfect structure). The authors considered the problem of undamped axial vibrations of a rod. Damage was modeled as a massless spring of infinitesimal length and unknown stiffness $\left(K_{a}\right)$. It was assumed that the mode shapes of the undamaged structure remain unchanged in the damaged state. The location of the damage and the value of $K_{a}$ were the unknowns to be determined using the observed frequency shifts. Liang et. al. [10] utilized a similar approach for the problem of flexural vibrations of an Euler-Bernoulli beam, where cracks were modeled using rotational springs. The authors plotted the values of normalized spring stiffness versus damage location for a few natural frequencies of the beam to identify the damage site. The methods would fail for components (e.g. a simply supported beam) for which symmetric damage locations would produce the same frequency shifts.

To improve the performance of frequency-based methods, mode shapes and improved crack models were included in the analysis. Rizos et. al. [15] presented such a method using the flexural vibrations of a cantilever beam. The crack was modeled by a massless rotational spring. Ideas from fracture mechanics were used to relate the spring stiffness to crack depth. The amplitudes of the induced motion at two locations on the beam were used to obtain 
the damage location and damage severity. Pandey et. al. [13] demonstrated a methodology based on changes in the curvature of mode shapes. Mode shapes for a damaged and undamaged beam were obtained using the finite element method. Vibrations of a simply supported and a cantilever beam were considered. It was found that curvature mode shapes were more sensitive to damage detection than displacement mode shapes. The method was helpful in determining a damage zone, but could not provide any quantitative estimate of the damage magnitude. Cao and Zimmerman [3] suggested load-dependent Ritz vectors extracted from the dynamic response data (Cao and Zimmerman [4]) as drivers for damage detection. Minimum rank perturbation theory was used to calculate the stiffness loss using measured eigenvectors and eigenfrequencies as an input. The presence and location of damage was determined in terms of a generalized damage residual; the Ritz vectors were found to be more sensitive to damage than modal vectors. Ruotolo and Surace [16] presented a method for damage identification in multiply-cracked beams by employing optimization techniques and genetic algorithms. Cerri and Vestroni [5] addressed the problem of diffused cracking in a beam. Two methods, based on characteristic equation error, and comparison between measured and analytical frequencies respectively, were discussed. Banici and Kallivokas [2] suggested a method for crack detection in beams utilizing multiple spectra. First-order perturbation approximations were employed to calculate the first few frequencies of the damaged beam. Crack locations and severities were obtained by minimizing the misfit between measured and computed eigenfrequencies. Solution mul- 
tiplicity was alleviated by using data from auxiliary experiments using small masses to modify the used spectra. Many of the aforementioned approaches lack the ability to quantify the damage magnitude, and, could not lead to quantification of remaining strength. Moreover, most modal parameter-based methods require calibration with the structure's virgin state. However, the ease of experimentation and the low computational cost make these techniques desirable, despite their shortcomings.

Among methods that do not rely on modal characteristics for assessment, the most prominent is the impact-echo method (Sansalone and Carino [17]). It uses stress waves to quantify the thickness of slabs, but also to detect cracks, primarily in concrete structures, and has been standardized by ASTM (ASTM C 1074) and ACI (ACI 228.1R). The technique is based on the reflection of P-waves. The damaged component is subjected to an impact and the resulting motion is recorded using a transducer. Either travel times or the frequency content of the time signal can be used to detect the presence and location of the flaw. The method was developed to test concrete pavements, slabs, or other plate-like structures. For example, the spectrum of an undamaged plate shows peak at the so-called plate thickness frequency, and that of a damaged plate presents a shift in the location of this peak. The depth at which the crack is located can be calculated using this shift. Similar to the methods mentioned earlier, the impact-echo is cumbersome for finding defects in frames or bridge decks, due to the difficulties in the interpretation of the collected response, owing to the multiple reflections/refractions (Sansalone 
and Streett [18]). The method provides only qualitative measurement of the damage, which is not directly useful in predicting response of the structure to any other applied load. It is, in general, more suitable for detecting cracks running parallel to a test surface than those running orthogonal to it.

On the other hand, the territory of direct time-domain-based inversion schemes remains, by and large, unexplored. In this work, we discuss a total wavefield-based inversion technique using a partial-differential-equation (PDE) constrained optimization approach for reconstructing the material properties of a flexural member or of complete frames. The proposed approach yields the spatial variability of a target property, leading to a better quantitative estimate, without requiring a priori knowledge of the undamaged state. It is equally effective in recovering smooth as well as sharply varying profiles, albeit computationally more expensive than the modal parameters-based algorithms discussed earlier.

The inversion process is inspired by similar developments in geophysical exploration, where full waveform approaches are increasingly favored. The inversion process is driven by the misfit between measured and computed responses to a known excitation (in the time-domain). Thus, the experimental setup is no different than what is currently used by other methods. The measured response is the measured time-history of the deflections at a few sensor locations in the damaged member, and contains information about the spatial variability of the material property we seek to determine. Reconstruction of the property's spatial variability will reveal any structural flaws, if 
present; it will also allow for the complete imaging of the probed structure. The computed response refers to the time-history of the component's deflections at the same locations as the recordings, calculated using an estimate of the sought property. The actual spatial distribution of the property is the one that makes the misfit identically zero. This condition is too strong to satisfy in practice, and hence we try to minimize the misfit functional, while satisfying the underlying physics, using the systematic framework of PDE-constrained optimization. Fulfillment of the pertinent physics is achieved by augmenting the misfit functional by the weak imposition of the governing PDEs, boundary and initial conditions, as well as of any continuity conditions (Lions [11]). For a convex functional, minimization corresponds to satisfying the first-order optimality conditions; convexity here is not ensured, and thus, instead, we seek to satisfy stationarity of the augmented functional. These conditions lead to time-dependent state and adjoint problems, and a time-independent control problem, which upon discretization lead to a classic KKT (KarushKuhn-Tucker) system. Numerical solutions of the state and adjoint problems are obtained using finite elements. We adopt a reduced-space scheme to solve the KKT system, and iteratively update the material properties until convergence. In general, uniqueness (or even existence) of the solution to the inverse problem cannot be guaranteed; to tackle solution multiplicity we use regularization schemes such as Tikhonov (TN) and Total Variation (TV). Normally, both these methods require a user-selected regularization factor as input: here, we use a regularization factor continuation scheme, which automatically selects 
the factor based on the current state of variables in the control problem.

This thesis is organized as follows: chapter 2 discusses the forward problem, i.e. the governing PDEs and boundary conditions as per the Timoshenko beam theory for modeling a $1 \mathrm{D}$ flexural member. In chapter 3 , we present preliminaries of the inverse problem and PDE-constrained optimization approach. This includes the Lagrangian functional, first order optimality conditions - state, adjoint and control problems along with regularization techniques. Chapter 4 describes the finite element formulation of the state and adjoint problems along with numerical integration schemes used for their solution. It also contains the general algorithm for inversion process. In chapter 5 , we report the results of our numerical experiments on a simply supported beam. We choose Young's modulus to be the inversion variable. Chapter 6 presents extensions of the inversion procedure for material profile reconstruction in frames and algorithmic modifications to accommodate the moment of inertia as the inversion variable. Conclusions are summarized in chapter 7 . 


\section{Chapter 2}

\section{The forward problem}

To fix ideas, we focus on the problem of undamped flexural vibrations of a simply-supported beam. In order to be able to capture localized damage, it is, in general, necessary that high frequencies (or high wavenumbers) be used for probing. This, in turn, necessitates the adoption of Timoshenko beams for modeling the flexural elements (Timoshenko [21,22]), since the usual EulerBernoulli theory leads to a non-physical dispersion relation, according to which high-frequency components travel almost instantaneously (Graff [6]). By contrast, the inclusion of rotary inertia and shear effects, per the Timoshenko assumptions, allows for a fairly accurate dispersion curve (when compared to three-dimensional solutions), and a physically faithful representation of flexu-

ral waves in the beam (Figure 2.1). Denoting the transverse displacement by $w(x)$, the slope due to bending by $\psi(x)$, and the contribution to the total slope due to shear deformation by $\gamma(x)$, the total bending slope, per the Timoshenko model, can be expressed as:

$$
\frac{d w(x)}{d x}=\psi(x)+\gamma(x)
$$

Then, the problem of the undamped vibrations of a Timoshenko beam, 

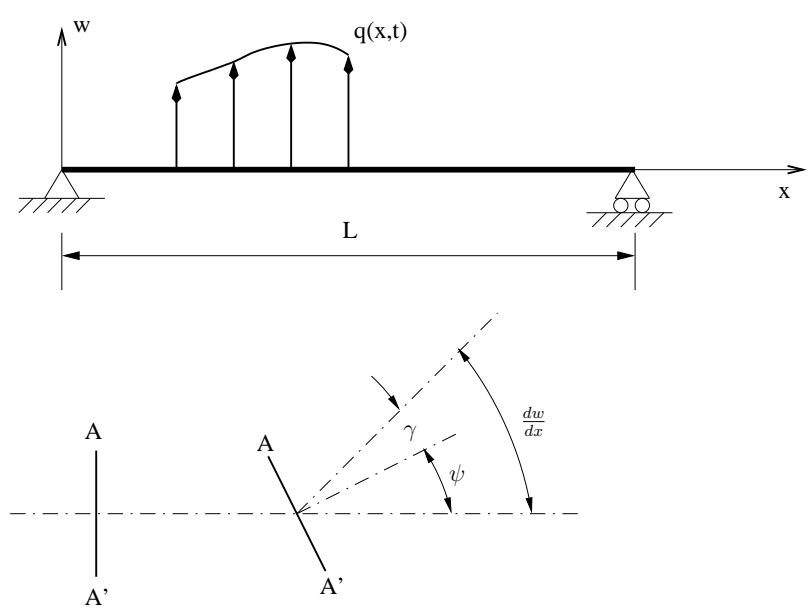

Figure 2.1: Schematic of Timoshenko beam: initially plane cross-section AA' remains plane post-deformation with shear effects taken into account

or forward problem, can be stated as: find $w(x, t)$ and $\psi(x, t)$, such that:

$$
\begin{gathered}
\frac{\partial}{\partial x}\left\{G A K_{s}\left(\frac{\partial w}{\partial x}-\psi\right)\right\}-\rho A \frac{\partial^{2} w}{\partial t^{2}}=-q, \\
G A K_{s}\left(\frac{\partial w}{\partial x}-\psi\right)+\frac{\partial}{\partial x}\left(E I \frac{\partial \psi}{\partial x}\right)-\rho I \frac{\partial^{2} \psi}{\partial t^{2}}=0,
\end{gathered}
$$

where $x$ denotes position, with $x \in(0, L)$, and $t$ denotes time, with $t \in(0, T]$ ( $T$ is the total observation time). $E \equiv E(x)$ is Young's modulus, $G \equiv G(x)$ is the shear modulus, $\rho \equiv \rho(x)$ is the mass density, and $A \equiv A(x), I \equiv I(x)$ denote the cross-sectional area and moment of inertia, respectively. $K_{s}$ denotes the Timoshenko shear factor, which depends on the cross-sectional geometry, and $q \equiv q(x, t)$ is the applied excitation. Either $E$ or $G$ can be eliminated from the above equations using $G=\frac{E}{2(1+\nu)}$, where $\nu$ is Poisson's ratio. Applicable boundary conditions can be chosen from Table 2.1 below. 


\begin{tabular}{|c|c|}
\hline Support Condition & Mathematical Representation \\
\hline Roller & $w=0, E I \frac{\partial \psi}{\partial x}=0$ \\
Hinge & $w=0, E I \frac{\partial \psi}{\partial x}=0$ \\
Fixed & $w=0, \psi=0$ \\
\hline
\end{tabular}

Table 2.1: Boundary conditions for Timoshenko beam (Huang [7])

Thus, for a simply supported beam, initially at rest, the following boundary and initial conditions apply:

Boundary conditions:

$$
\begin{aligned}
w(0, t) & =0 \quad, \quad w(L, t)=0 \\
\left(E I \frac{\partial \psi}{\partial x}\right)_{x=0} & =0 \quad, \quad\left(E I \frac{\partial \psi}{\partial x}\right)_{x=L}=0 .
\end{aligned}
$$

Initial conditions:

$$
\begin{aligned}
w(x, 0) & =0, & \psi(x, 0) & =0, \\
\frac{\partial w}{\partial t}(x, 0) & =0, & \frac{\partial \psi}{\partial t}(x, 0) & =0 .
\end{aligned}
$$

Equations (2.2) through (2.7) completely define the initial boundary value problem (IBVP) or the forward problem. 


\section{Chapter 3}

\section{The inverse problem}

\subsection{Objective functional}

Without loss of generality, we select the Young's modulus $E(x)$ as the inversion variable, whose spatial distribution we seek to determine; in chapter 6 we discuss other possible choices. We seek to minimize the misfit functional $\mathcal{J}$, in the least-squares sense, between computed and measured deflections, subject to the physics implied by the forward problem. To alleviate the inherent solution multiplicity we also augment the misfit $\mathcal{J}$ by a regularization term $\mathcal{R}(E)$ to arrive at an objective functional $\mathcal{F}$. Thus, we seek to minimize:

$$
\begin{aligned}
\mathcal{F} & =\mathcal{J}+\mathcal{R}(E) \\
& =\frac{1}{2} \sum_{i=1}^{N} \int_{0}^{T}\left[w^{m}\left(x_{i}, t\right)-w\left(x_{i}, t\right)\right]^{2} \mathrm{~d} t+\mathcal{R}(E),
\end{aligned}
$$

subject to (2.2)-(2.7). In equation (3.1), $x_{i}$ denotes the location of the $i$-th sensor, and $N$ denotes the total number of sensors used. $w^{m}(x, t)$ and $w(x, t)$ represent the measured and computed responses, respectively, with $w(x, t)$ corresponding to an assumed profile $E(x)$. 


\subsubsection{Regularization}

The misfit functional contains incomplete information about the dynamic response of the probed component(s), due to the fact that, in practice, only a few sensors are used to measure the response. Thus, the inverse problem that is based solely on the misfit functional suffers from non-uniqueness of the solutions for $E(x)$. The difficulty is commonly alleviated by enforcing an additional constraint in the form of regularization. Two types of regularization schemes are used in this work, namely, Tikhonov (TN) regularization, and Total Variation (TV) regularization.

Tikhonov regularization is one of the most frequently used regularization schemes (Tikhonov [20]). It is defined as the $L^{2}$-norm of the gradient of the inversion variable; accordingly, and for the one-dimensional Timoshenko problem of interest here, the last term in (3.1) assumes the form:

$$
\mathcal{R}^{T N}(E)=\frac{R_{E}}{2} \int_{0}^{L}\left(\frac{d E}{d x}\right)^{2} \mathrm{~d} x,
$$

where $R_{E}$ is the regularization factor -a user-defined scalar constant that weighs the penalty imposed by the regularization term. In general, TN regularization tends to smoothen sharp variations of the inversion variable, as betrayed by the derivative term in (3.2). By contrast, Total Variation regularization, in general, allows for easier recovery of discontinuities, due to the presence of a semi-norm in its definition, as in:

$$
\mathcal{R}^{T V}(E)=R_{E} \int_{0}^{L}\left[\left(\frac{d E}{d x}\right)^{2}+\epsilon\right]^{\frac{1}{2}} \mathrm{~d} x
$$


where $\epsilon$ is a small positive constant (e.g. $10^{-6}$ for applications). We remark that the choice of the regularization factor is critical for the performance of either the $\mathrm{TN}$ or the TV scheme. In chapter 4 , we discuss a regularization factor continuation scheme that aids in the convergence of the inversion process.

\subsection{PDE-constrained optimization}

\subsubsection{Lagrangian functional}

PDE-constrained optimization approach is to be employed for minimization of the objective functional in (3.1), subject to (2.2) to (2.7). To this end, the inverse problem is reformulated as an unconstrained optimization problem by weakly imposing the underlying constraints $(2.2)$ to $(2.7)$ on the objective functional $\mathcal{F}$ in $(3.1)$ to obtain the Lagrangian functional $-\mathcal{L}$.

$$
\begin{aligned}
& \mathcal{L}\left(w, \psi, \lambda_{w}, \lambda_{\psi}, \lambda_{B 1}, \lambda_{B 2} \lambda_{I 1}, \lambda_{I 2}, E\right) \\
= & \frac{1}{2} \sum_{i=1}^{N} \int_{0}^{T}\left[w^{m}\left(x_{i}, t\right)-w\left(x_{i}, t\right)\right]^{2} \mathrm{~d} t+\mathcal{R}(E) \\
& +\int_{0}^{T} \int_{0}^{L} \lambda_{w}\left[\frac{\partial}{\partial x}\left\{G A K_{s}\left(\frac{\partial w}{\partial x}-\psi\right)\right\}-\rho A \frac{\partial^{2} w}{\partial t^{2}}+q\right] \mathrm{d} x \mathrm{~d} t \\
& +\int_{0}^{T} \int_{0}^{L} \lambda_{\psi}\left[G A K_{s}\left(\frac{\partial w}{\partial x}-\psi\right)+\frac{\partial}{\partial x}\left(E I \frac{\partial \psi}{\partial x}\right)-\rho I \frac{\partial^{2} \psi}{\partial t^{2}}\right] \mathrm{d} x \mathrm{~d} t \\
& +\int_{0}^{T} \lambda_{B 1}\left[E I \frac{\partial \psi}{\partial x}\right]_{x=0}+\lambda_{B 2}\left[E I \frac{\partial \psi}{\partial x}\right]_{x=L} \mathrm{~d} t \\
& +\int_{0}^{L} \lambda_{I 1}\left[\frac{\partial w}{\partial t}\right]_{t=0}+\lambda_{I 2}\left[\frac{\partial \psi}{\partial t}\right]_{t=0} \mathrm{~d} x,
\end{aligned}
$$

where only Neumann-type conditions have been imposed (Dirichlet conditions 
will be explicitly enforced), and $\lambda_{w}, \lambda_{\psi}, \lambda_{B 1}, \lambda_{B 2}, \lambda_{I 1}, \lambda_{I 2}$ denote the Lagrange multipliers (or adjoint variables). We seek a stationary point for $\mathcal{L}$, by requiring the satisfaction of the first-order optimality conditions. Specifically, we require that the first variation of $\mathcal{L}$ with respect to the state variables $(w, \psi)$, the Lagrange multipliers $(\lambda)$, and the control variable $(E)$, vanish. This, in turn, gives rise to state, adjoint, and control problems, as discussed in the following sections.

\subsubsection{The first optimality condition}

We require the vanishing of the first variation of the Lagrangian functional with respect to the Lagrange multipliers (or adjoint variables):

$$
\begin{gathered}
\delta_{\lambda_{w}} \mathcal{L}=0, \delta_{\lambda_{\psi}} \mathcal{L}=0, \delta_{\lambda_{B 1}} \mathcal{L}=0, \\
\delta_{\lambda_{B 2}} \mathcal{L}=0, \delta_{\lambda_{I 1}} \mathcal{L}=0, \delta_{\lambda_{I 2}} \mathcal{L}=0 .
\end{gathered}
$$

It is easily verifiable that equations (3.5) yield the state problem, which is identical to the forward problem (2.2)-(2.7).

State Problem:

$$
\begin{gathered}
\frac{\partial}{\partial x}\left\{G A K_{s}\left(\frac{\partial w}{\partial x}-\psi\right)\right\}-\rho A \frac{\partial^{2} w}{\partial t^{2}}=-q, \\
G A K_{s}\left(\frac{\partial w}{\partial x}-\psi\right)+\frac{\partial}{\partial x}\left(E I \frac{\partial \psi}{\partial x}\right)-\rho I \frac{\partial^{2} \psi}{\partial t^{2}}=0,
\end{gathered}
$$

for $x \in(0, L), t \in(0, T]$. 
Boundary conditions:

$$
\begin{aligned}
w(0, t) & =0 \\
w(L, t) & =0, \\
\left(E I \frac{\partial \psi}{\partial x}\right)_{x=0} & =0, \\
\left(E I \frac{\partial \psi}{\partial x}\right)_{x=L} & =0 .
\end{aligned}
$$

Initial conditions:

$$
\begin{aligned}
w(x, 0) & =0, \\
\psi(x, 0) & =0, \\
\frac{\partial w}{\partial t}(x, 0) & =0, \\
\frac{\partial w}{\partial t}(x, 0) & =0 .
\end{aligned}
$$

\subsubsection{The second optimality condition}

Vanishing of the first variation of the Lagrangian with respect to the state variables - $(w, \psi)$, i.e.:

$$
\begin{aligned}
& \delta_{w} \mathcal{L}=0, \\
& \delta_{\psi} \mathcal{L}=0 .
\end{aligned}
$$


give rise to the adjoint problem. We note that displacement boundary conditions and initial conditions imply:

$$
\begin{aligned}
& \delta w(0, t)=0, \\
& \delta w(L, t)=0, \\
& \delta \psi(x, 0)=0, \\
& \delta w(x, 0)=0, \\
& \frac{\partial \delta \psi(x, 0)}{\partial t}=0, \\
& \frac{\partial \delta w(x, 0)}{\partial t}=0 . \\
& \delta_{w} \mathcal{L}=\sum_{i=1}^{N} \int_{0}^{T} \int_{0}^{L}-\left(w^{m}-w\right) \delta\left(x-x_{i}\right) \delta w \mathrm{~d} x \mathrm{~d} t \\
&+\int_{0}^{T} \int_{0}^{L} \lambda_{w}\left[\frac{\partial}{\partial x}\left\{G A K_{s}\left(\frac{\partial \delta w}{\partial x}\right)\right\}-\rho A \frac{\partial^{2} \delta w}{\partial t^{2}}\right] \mathrm{d} x \mathrm{~d} t \\
&+\int_{0}^{T} \int_{0}^{L} \lambda_{\psi}\left[G A K_{s}\left(\frac{\partial \delta w}{\partial x}\right)\right] \mathrm{d} x \mathrm{~d} t .
\end{aligned}
$$

We simplify the above expression term-by-term. Let,

$$
\begin{aligned}
\alpha= & \int_{0}^{T} \int_{0}^{L} \lambda_{w} \frac{\partial}{\partial x}\left\{G A K_{s}\left(\frac{\partial \delta w}{\partial x}\right)\right\} \mathrm{d} x \mathrm{~d} t \\
= & \int_{0}^{T}\left[\lambda_{w} G A K_{s} \frac{\partial \delta w}{\partial x}\right]_{0}^{L}-\left[\int_{0}^{L} G A K_{s} \frac{\partial \delta w}{\partial x} \frac{\partial \lambda_{w}}{\partial x} \mathrm{~d} x\right] \mathrm{d} t \\
= & \int_{0}^{T}\left[\lambda_{w} G A K_{s} \frac{\partial \delta w}{\partial x}\right]_{0}^{L} \mathrm{~d} t \\
& -\int_{0}^{T}\left[G A K_{s} \frac{\partial \lambda_{w}}{\partial x} \delta w\right]_{0}^{L}-\left[\int_{0}^{L} \delta w \frac{\partial}{\partial x}\left(G A K_{s} \frac{\partial \lambda_{w}}{\partial x}\right) \mathrm{d} x\right] \mathrm{d} t .
\end{aligned}
$$


Using equation (3.17) and (3.18), we get

$$
\begin{gathered}
\delta w(0, t)=0 \\
\delta w(L, t)=0 . \\
\therefore \alpha=\int_{0}^{T}\left[\lambda_{w} G A K_{s} \frac{\partial \delta w}{\partial x}\right]_{0}^{L}+\int_{0}^{L} \delta w \frac{\partial}{\partial x}\left(G A K_{s} \frac{\partial \lambda_{w}}{\partial x}\right) \mathrm{d} x \mathrm{~d} t .
\end{gathered}
$$

Let,

$$
\begin{gathered}
\beta=-\int_{0}^{T} \int_{0}^{L} \lambda_{w} \rho A \frac{\partial^{2} \delta w}{\partial t^{2}} \mathrm{~d} x \mathrm{~d} t \\
=-\int_{0}^{L}\left[\lambda_{w} \rho A \frac{\partial \delta w}{\partial t}\right]_{0}^{T}-\left[\int_{0}^{T} \rho A \frac{\partial \delta w}{\partial t} \frac{\partial \lambda_{w}}{\partial t} \mathrm{~d} t\right] \mathrm{d} x . \\
\lambda_{w}(x, T)=0 .
\end{gathered}
$$

From equation (3.22),

$$
\begin{aligned}
& \frac{\partial \delta w}{\partial t}(x, 0)=0 . \\
& \therefore \beta=\int_{0}^{L} \int_{0}^{T} \rho A \frac{\partial \delta w}{\partial t} \frac{\partial \lambda_{w}}{\partial t} \mathrm{~d} t \mathrm{~d} x \\
&=\int_{0}^{t}\left[\rho A \frac{\partial \lambda_{w}}{\partial t} \delta w\right]_{0}^{T}-\left[\int_{0}^{T} \rho A \delta w \frac{\partial^{2} \lambda_{w}}{\partial t^{2}} \mathrm{~d} t\right] \mathrm{d} x . \\
& \frac{\partial \lambda_{w}}{\partial t}(x, T)=0 \\
& \delta w(x, 0)=0 .
\end{aligned}
$$




$$
\therefore \beta=-\int_{0}^{L} \int_{0}^{T} \rho A \delta w \frac{\partial^{2} \lambda_{w}}{\partial t^{2}} \mathrm{~d} t \mathrm{~d} x .
$$

Let,

$$
\begin{aligned}
\Gamma & =\int_{0}^{T} \int_{0}^{L} \lambda_{\psi}\left\{G A K_{s}\left(\frac{\partial \delta w}{\partial x}\right)\right\} \mathrm{d} x \mathrm{~d} t \\
& =\int_{0}^{T}\left[\lambda_{\psi} G A K_{s} \delta w\right]_{0}^{L}-\left[\int_{0}^{L} \delta w \frac{\partial}{\partial x}\left(G A K_{s} \lambda_{\psi}\right) \mathrm{d} x\right] \mathrm{d} t
\end{aligned}
$$

From equations (3.17) and (3.18), we have

$$
\begin{gathered}
\delta w(0, t)=0 \\
\delta w(L, t)=0 . \\
\therefore \Gamma=-\int_{0}^{T} \int_{0}^{L} \delta w \frac{\partial}{\partial x}\left(G A K_{s} \lambda_{\psi}\right) \mathrm{d} x \mathrm{~d} t .
\end{gathered}
$$

Substituting values of $\alpha, \beta, \Gamma$ in equation(3.23), we get

$$
\begin{aligned}
\delta_{w} \mathcal{L}= & 0 \\
= & \int_{0}^{T} \int_{0}^{L}\left[\frac{\partial}{\partial x}\left[G A K_{s}\left(\frac{\partial \lambda_{w}}{\partial x}-\lambda_{\psi}\right)\right]-\rho A \frac{\partial^{2} \lambda_{w}}{\partial t^{2}}\right. \\
& \left.-\sum_{i=1}^{N}\left(w^{m}-w\right) \delta\left(x-x_{i}\right)\right] \delta w(x, t) \mathrm{d} x \mathrm{~d} t \\
& +\int_{0}^{T}\left[\lambda_{w}(L, t) G(L) A(L) K_{s}(L)\right]\left[\frac{\partial \delta w}{\partial x}\right]_{x=L} \mathrm{~d} t \\
& -\int_{0}^{T}\left[\lambda_{w}(0, t) G(0) A(0) K_{s}(0)\right]\left[\frac{\partial \delta w}{\partial x}\right]_{x=0} \mathrm{~d} t .
\end{aligned}
$$


The function $\delta w(x, t)$ for $x \in(0, L)$ is arbitrary and values of $\frac{\partial \delta w}{\partial x}$ at $x=0$ and $x=L$ are arbitrary. Hence, their multipliers in equation (3.28) are identically zero. Thus, $\delta_{w} \mathcal{L}=0$ implies that,

$$
\begin{gathered}
\frac{\partial}{\partial x}\left[G A K_{s}\left(\frac{\partial \lambda_{w}}{\partial x}-\lambda_{\psi}\right)\right]-\rho A \frac{\partial^{2} \lambda_{w}}{\partial t^{2}}=\sum_{i=1}^{N} \int_{0}^{L}\left(w_{i}^{m}-w_{i}\right) \mathrm{d} t \\
\lambda_{w}(0, t)=0 \\
\lambda_{w}(L, t)=0 \\
\delta_{\psi} \mathcal{L}=\int_{0}^{T} \int_{0}^{L}-\lambda_{w} \frac{\partial}{\partial x}\left(G A K_{s} \delta \psi\right) \mathrm{d} x \mathrm{~d} t \\
+\int_{0}^{T} \int_{0}^{L} \lambda_{\psi}\left[\frac{\partial}{\partial x}\left\{E I\left(\frac{\partial \delta \psi}{\partial x}\right)\right\}-G A K_{s} \delta \psi-\rho I \frac{\partial^{2} \delta \psi}{\partial t^{2}}\right] \mathrm{d} x \mathrm{~d} t \\
+\int_{0}^{T} \lambda_{B 1}\left[E I \frac{\partial \delta \psi}{\partial x}\right]_{x=0}+\lambda_{B 2}\left[E I \frac{\partial \delta \psi}{\partial x}\right]_{x=L} \mathrm{~d} t .
\end{gathered}
$$

We simplify the above expression term-by-term.

Let,

$$
\begin{aligned}
\epsilon & =-\int_{0}^{T} \int_{0}^{L} \lambda_{w} \frac{\partial}{\partial x}\left\{G A K_{s} \delta \psi\right\} \mathrm{d} x \mathrm{~d} t \\
& =-\int_{0}^{T}\left[\lambda_{w} G A K_{s} \delta \psi\right]_{0}^{L}-\left[\int_{0}^{L} G A K_{s} \delta \psi \frac{\partial \lambda_{w}}{\partial x} \mathrm{~d} x\right] \mathrm{d} t .
\end{aligned}
$$

From equations (3.30) and (3.31), we have

$$
\begin{aligned}
& \lambda_{w}(0, t)=0, \\
& \lambda_{w}(L, t)=0 .
\end{aligned}
$$




$$
\therefore \epsilon=\int_{0}^{T} \int_{0}^{L} \delta \psi G A K_{s} \frac{\partial \lambda_{w}}{\partial x} \mathrm{~d} x \mathrm{~d} t .
$$

Let,

$$
\begin{aligned}
\eta= & \int_{0}^{T} \int_{0}^{L} \lambda_{\psi} \frac{\partial}{\partial x}\left\{E I\left(\frac{\partial \delta \psi}{\partial x}\right)\right\} \mathrm{d} x \mathrm{~d} t \\
= & \int_{0}^{T}\left[\lambda_{\psi} E I \frac{\partial \delta \psi}{\partial x}\right]_{0}^{L}-\left[\int_{0}^{L} E I \frac{\partial \delta \psi}{\partial x} \frac{\partial \lambda_{\psi}}{\partial x} \mathrm{~d} x\right] \mathrm{d} t \\
= & -\int_{0}^{T}\left[E I \frac{\partial \lambda_{\psi}}{\partial x} \delta \psi\right]_{0}^{L}-\left[\int_{0}^{L} \delta \psi \frac{\partial}{\partial x}\left(E I \frac{\partial \lambda_{\psi}}{\partial x}\right) \mathrm{d} x\right] \mathrm{d} t \\
& +\int_{0}^{T}\left[\lambda_{\psi} E I \frac{\partial \delta \psi}{\partial x}\right]_{0}^{L} \mathrm{~d} t \\
= & \int_{0}^{T} \int_{0}^{L} \delta \psi \frac{\partial}{\partial x}\left(E I \frac{\partial \lambda_{\psi}}{\partial x}\right) \mathrm{d} x \mathrm{~d} t \\
& +\int_{0}^{T}\left[\lambda_{\psi} E I \frac{\partial \delta \psi}{\partial x}\right]_{0}^{L}-\left[E I \frac{\partial \lambda_{\psi}}{\partial x} \delta \psi\right]_{0}^{L} \mathrm{~d} t .
\end{aligned}
$$

Let,

$$
\begin{gathered}
\zeta=-\int_{0}^{T} \int_{0}^{L} \lambda_{\psi} \rho I \frac{\partial^{2} \delta \psi}{\partial t^{2}} \mathrm{~d} x \mathrm{~d} t \\
=-\int_{0}^{L}\left[\lambda_{\psi} \rho I \frac{\partial \delta \psi}{\partial t}\right]_{0}^{T}-\left[\int_{0}^{T} \rho I \frac{\partial \delta \psi}{\partial t} \frac{\partial \lambda_{\psi}}{\partial t} \mathrm{~d} t\right] \mathrm{d} x . \\
\lambda_{\psi}(x, T)=0 .
\end{gathered}
$$

From equation (3.21), we get

$$
\begin{gathered}
\frac{\partial \delta \psi(x, 0)}{\partial t}=0 . \\
\therefore \zeta=\int_{0}^{L} \int_{0}^{T} \rho I \frac{\partial \delta \psi}{\partial t} \frac{\partial \lambda_{\psi}}{\partial t} \mathrm{~d} t \mathrm{~d} x \\
=\int_{0}^{L}\left[\rho I \frac{\partial \lambda_{\psi}}{\partial t} \delta \psi\right]_{0}^{T}-\left[\int_{0}^{T} \rho I \delta \psi \frac{\partial^{2} \lambda_{\psi}}{\partial t^{2}} \mathrm{~d} t\right] \mathrm{d} x .
\end{gathered}
$$




$$
\frac{\partial \lambda_{\psi}}{\partial t}(x, T)=0 .
$$

From equation (3.19), we get

$$
\begin{gathered}
\delta \psi(x, 0)=0 . \\
\therefore \zeta=-\int_{0}^{L} \int_{0}^{T} \rho I \delta \psi \frac{\partial^{2} \lambda_{\psi}}{\partial t^{2}} \mathrm{~d} t \mathrm{~d} x .
\end{gathered}
$$

Substituting values of $\epsilon, \eta, \zeta$ in equation(3.32), we get

$$
\begin{aligned}
\delta_{\psi} \mathcal{L}= & 0 \\
= & \int_{0}^{T} \int_{0}^{L}\left[\frac{\partial}{\partial x}\left[E I \frac{\partial \lambda_{\psi}}{\partial x}\right]+G A K_{s}\left(\frac{\partial \lambda_{w}}{\partial x}-\lambda_{\psi}\right)\right. \\
& \left.\quad-\rho I \frac{\partial^{2} \lambda_{\psi}}{\partial t^{2}}\right] \delta \psi(x, t) \mathrm{d} x \mathrm{~d} t \\
+ & \int_{0}^{T}\left[\lambda_{\psi} E I \frac{\partial \delta \psi}{\partial x}\right]_{0}^{L}-\left[E I \frac{\partial \lambda_{\psi}}{\partial x} \delta \psi\right]_{0}^{L} \mathrm{~d} t \\
& +\int_{0}^{T} \lambda_{B 1}\left[E I \frac{\partial \delta \psi}{\partial x}\right]_{x=0}+\lambda_{B 2}\left[E I \frac{\partial \delta \psi}{\partial x}\right]_{x=L} \mathrm{~d} t .
\end{aligned}
$$


Thus,

$$
\begin{aligned}
\delta_{\psi} \mathcal{L}= & 0 \\
= & \int_{0}^{T} \int_{0}^{L}\left[\frac{\partial}{\partial x}\left[E I \frac{\partial \lambda_{\psi}}{\partial x}\right]+G A K_{s}\left(\frac{\partial \lambda_{w}}{\partial x}-\lambda_{\psi}\right)\right. \\
& \left.\quad-\rho I \frac{\partial^{2} \lambda_{\psi}}{\partial t^{2}}\right] \delta \psi(x, t) \mathrm{d} x \mathrm{~d} t \\
& -\int_{0}^{T}\left[E I \frac{\partial \lambda_{\psi}}{\partial x}\right]_{x=L} \delta \psi(L, t)-\left[E I \frac{\partial \lambda_{\psi}}{\partial x}\right]_{x=0} \delta \psi(0, t) \mathrm{d} t \\
+ & \int_{0}^{T}\left[E(L) I(L) \lambda_{\psi}(L, t)+\lambda_{B 2} E(L) I(L)\right]\left[\frac{\partial \delta \psi}{\partial x}\right]_{x=L} \\
& -\left[E(0) I(0) \lambda_{\psi}(0, t)-\lambda_{B 1} E(0) I(0)\right]\left[\frac{\partial \delta \psi}{\partial x}\right]_{x=0} \mathrm{~d} t .
\end{aligned}
$$

The following quantities are arbitrary:

$$
\begin{aligned}
\delta \psi(x, t) \quad & \text { for } \quad x \in(0, L), \\
& \delta \psi(L, t), \\
& \delta \psi(0, t), \\
& {\left[\frac{\partial \delta \psi}{\partial x}\right]_{x=L}, } \\
& {\left[\frac{\partial \delta \psi}{\partial x}\right]_{x=0} . }
\end{aligned}
$$

There results:

$$
\begin{gathered}
G A K_{s}\left(\frac{\partial \lambda_{w}}{\partial x}-\lambda_{\psi}\right)+\frac{\partial}{\partial x}\left[E I \frac{\partial \lambda_{\psi}}{\partial x}\right]-\rho I \frac{\partial^{2} \lambda_{\psi}}{\partial t^{2}}=0 \\
\left(E I \frac{\partial \lambda_{\psi}}{\partial x}\right)_{x=0}=0 \\
\left(E I \frac{\partial \lambda_{\psi}}{\partial x}\right)_{x=L}=0
\end{gathered}
$$




$$
\begin{aligned}
& \lambda_{B 1}(t)=\lambda_{\psi}(0, t), \\
& \lambda_{B 2}(t)=-\lambda_{\psi}(L, t) .
\end{aligned}
$$

Based on equations (3.29) to (3.31) and (3.38) to (3.42) above, the adjoint problem can be summarized as follows: Adjoint Problem

$$
\begin{gathered}
\frac{\partial}{\partial x}\left[G A K_{s}\left(\frac{\partial \lambda_{w}}{\partial x}-\lambda_{\psi}\right)\right]-\rho A \frac{\partial^{2} \lambda_{w}}{\partial t^{2}}=\sum_{i=1}^{N}\left[w^{m}\left(x_{i}, t\right)-w\left(x_{i}, t\right)\right] \\
G A K_{s}\left(\frac{\partial \lambda_{w}}{\partial x}-\lambda_{\psi}\right)+\frac{\partial}{\partial x}\left[E I \frac{\partial \lambda_{\psi}}{\partial x}\right]-\rho I \frac{\partial^{2} \lambda_{\psi}}{\partial t^{2}}=0
\end{gathered}
$$

for $x \in(0, L)$, and $t \in[0, T)$.

Boundary Conditions:

$$
\begin{aligned}
\lambda_{w}(0, t) & =0, \\
\lambda_{w}(L, t) & =0 \\
\left(E I \frac{\partial \lambda_{\psi}}{\partial x}\right)_{x=0} & =0 \\
\left(E I \frac{\partial \lambda_{\psi}}{\partial x}\right)_{x=L} & =0 .
\end{aligned}
$$

Final Conditions:

$$
\begin{aligned}
\lambda_{w}(x, T) & =0, \\
\lambda_{\psi}(x, T) & =0, \\
\frac{\partial \lambda_{w}}{\partial t}(x, T) & =0, \\
\frac{\partial \lambda_{\psi}}{\partial t}(x, T) & =0 .
\end{aligned}
$$


We remark that, as is evident by (2.2)-(2.3) and (3.43)-(3.44), the state and adjoint problems are governed by identical operators, which greatly facilitates the numerical implementation and alleviates the computational cost. Notice further that, whereas the state problem is driven by the applied excitation, the adjoint problem is driven by the misfit between the measured and computed responses (right-hand-side of (3.43)). Moreover, the state problem is an initial value problem, whereas the adjoint problem is a final value problem.

\subsubsection{The third optimality condition:}

Finally, we demand that the first variation of the Lagrangian $-\mathcal{L}$ with respect to the control variable - $E$ should be equal to zero, or:

$$
\delta_{E} \mathcal{L}=0,
$$

which gives rise to the control problem. The form of the control problem depends on the type of regularization used. We derive the control equation for TN regularization below.

$$
\begin{aligned}
\delta_{E} \mathcal{L}= & R_{E} \int_{0}^{L}\left(\frac{d E}{d x}\right)\left(\frac{d \delta E}{d x}\right) \mathrm{d} x \\
& +\int_{0}^{T} \int_{0}^{L} \lambda_{w} \frac{\partial}{\partial x}\left[\frac{A K_{s}}{2(1+\nu)} \delta E\left(\frac{\partial w}{\partial x}-\psi\right)\right] \mathrm{d} x \mathrm{~d} t \\
& +\int_{0}^{T} \int_{0}^{L} \lambda_{\psi}\left[\frac{\partial}{\partial x}\left\{I \delta E\left(\frac{\partial \psi}{\partial x}\right)\right\}+\frac{A K_{s}}{2(1+\nu)} \delta E\left(\frac{\partial w}{\partial x}-\psi\right)\right] \mathrm{d} x \mathrm{~d} t \\
& +\int_{0}^{T} \lambda_{B 1}\left[I \delta E \frac{\partial \psi}{\partial x}\right]_{x=0}+\lambda_{B 2}\left[I \delta E \frac{\partial \psi}{\partial x}\right]_{x=L} \mathrm{~d} t .
\end{aligned}
$$


Let,

$$
\begin{aligned}
\xi & =R_{E} \int_{0}^{L}\left(\frac{d E}{d x}\right)\left(\frac{d \delta E}{d x}\right) \mathrm{d} x \\
& =R_{E}\left[\frac{d E}{d x} \delta E\right]_{0}^{L}-R \int_{0}^{L} \frac{d^{2} E}{d x^{2}} \delta E \mathrm{~d} x \\
& =-R_{E} \int_{0}^{L}\left[\frac{d E}{d x} \delta(x-0)-\frac{d E}{d x} \delta(x-L)+\frac{d^{2} E}{d x^{2}}\right] \delta E \mathrm{~d} x
\end{aligned}
$$

Let,

$$
\begin{aligned}
\chi= & \int_{0}^{T} \int_{0}^{L} \lambda_{w} \frac{\partial}{\partial x}\left[\frac{A K_{s}}{2(1+\nu)} \delta E\left(\frac{\partial w}{\partial x}-\psi\right)\right] \mathrm{d} x \mathrm{~d} t \\
= & \int_{0}^{T}\left[\lambda_{w} \frac{A K_{s}}{2(1+\nu)} \delta E\left(\frac{\partial w}{\partial x}-\psi\right)\right]_{0}^{L} \mathrm{~d} t \\
& -\int_{0}^{T} \int_{0}^{L} \frac{A K_{s}}{2(1+\nu)} \frac{\partial \lambda_{w}}{\partial x}\left(\frac{\partial w}{\partial x}-\psi\right) \delta E \mathrm{~d} x \mathrm{~d} t \\
= & -\int_{0}^{T} \int_{0}^{L} \frac{A K_{s}}{2(1+\nu)} \frac{\partial \lambda_{w}}{\partial x}\left(\frac{\partial w}{\partial x}-\psi\right) \delta E \mathrm{~d} x \mathrm{~d} t .
\end{aligned}
$$

Let,

$$
\begin{aligned}
\kappa & =\int_{0}^{T} \int_{0}^{L} \lambda_{\psi} \frac{\partial}{\partial x}\left\{I \delta E\left(\frac{\partial \psi}{\partial x}\right)\right\} \mathrm{d} x \mathrm{~d} t \\
& =\int_{0}^{T}\left[\lambda_{\psi} I \delta E \frac{\partial \psi}{\partial x}\right]_{0}^{L}-\left[\int_{0}^{L} I \frac{\partial \psi}{\partial x} \frac{\partial \lambda_{\psi}}{\partial x} \delta E \mathrm{~d} x\right] \mathrm{d} t \\
& =-\int_{0}^{T} \int_{0}^{L} I \frac{\partial \psi}{\partial x} \frac{\partial \lambda_{\psi}}{\partial x} \delta E \mathrm{~d} x \mathrm{~d} t
\end{aligned}
$$


Collecting the final expressions for $\xi, \chi$ and $\kappa$, we get,

$$
\begin{aligned}
\delta_{E} \mathcal{L}= & -R_{E}\left[\frac{d E(0)}{d x} \delta E(0)-\frac{d E(L)}{d x} \delta E(L)\right]-R_{E} \int_{0}^{L} \frac{d^{2} E}{d x^{2}} \delta E \mathrm{~d} x \\
& -\int_{0}^{T} \int_{0}^{L}\left[\frac{A K_{s}}{2(1+\nu)}\left(\frac{\partial w}{\partial x}-\psi\right)\left(\frac{\partial \lambda_{w}}{\partial x}-\lambda_{\psi}\right)\right. \\
& \left.\quad+I \frac{\partial \psi}{\partial x} \frac{\partial \lambda_{\psi}}{\partial x}\right] \delta E \mathrm{~d} x \mathrm{~d} t \\
= & 0 .
\end{aligned}
$$

Again, we use the arbitrariness of $\delta E(0)$ and $\delta E(L)$ to arrive at the control equations for both TN and TV type regularizations, stated below.

Control Problem - TN scheme:

$$
\begin{aligned}
& \delta_{E} \mathcal{L}=-\int_{0}^{L}\left\{R_{E} \frac{d^{2} E}{d x^{2}}\right. \\
&+\int_{0}^{T}\left[\frac{A K_{s}}{2(1+\nu)}\left(\frac{\partial w}{\partial x}-\psi\right)\left(\frac{\partial \lambda_{w}}{\partial x}-\lambda_{\psi}\right)\right. \\
&\left.\left.+I \frac{\partial \psi}{\partial x} \frac{\partial \lambda_{\psi}}{\partial x}\right] \mathrm{~d} t\right\} \delta E \mathrm{~d} x=0,
\end{aligned}
$$

subject to $\left(\frac{d E}{d x}\right)_{x=0}=0$ and $\left(\frac{d E}{d x}\right)_{x=L}=0$.

Control Problem - TV scheme:

$$
\begin{aligned}
\delta_{E} \mathcal{L}=-\int_{0}^{L} & \left\{R_{E} \epsilon \frac{d^{2} E}{d x^{2}}\left[\left(\frac{d E}{d x}\right)^{2}+\epsilon\right]^{-\frac{3}{2}}\right. \\
+ & \int_{0}^{T}\left[\frac{A K_{s}}{2(1+\nu)}\left(\frac{\partial w}{\partial x}-\psi\right)\left(\frac{\partial \lambda_{w}}{\partial x}-\lambda_{\psi}\right)\right. \\
+ & \left.\left.I \frac{\partial \psi}{\partial x} \frac{\partial \lambda_{\psi}}{\partial x}\right] \mathrm{~d} t\right\} \delta E \mathrm{~d} x=0,
\end{aligned}
$$

subject also to $\left(\frac{d E}{d x}\right)_{x=0}=0$ and $\left(\frac{d E}{d x}\right)_{x=L}=0$. 
We note that the control equations are time-independent. Stationarity of the Lagrangian functional is achieved when a set of variables - $\{w(x, t), \psi(x, t)$, $\left.\lambda_{w}(x, t), \lambda_{\psi}(x, t), E(x)\right\}$, satisfying the state, adjoint, and control problems is obtained. We discuss next the inversion scheme that leads to the reconstruction of $E(x)$. 


\section{Chapter 4}

\section{The inversion process}

There are at least two schemes to resolve the triplet of the state, adjoint, and control problems. In a full-space method, all three problems are solved simultaneously, resulting in increased computational cost. In a reduced-space method, the three problems are solved in sequence: firstly the time-dependent state problem is solved to yield the state variables (deflections $w(x, t)$, and slopes $\psi(x, t))$ for an assumed distribution of Young's modulus $E(x)$. Then, the time-dependent adjoint problem is solved driven by the misfit, to yield the adjoint variables $\lambda_{w}(x, t)$ and $\lambda_{\psi}(x, t)$. As will be discussed, standard finite elements are used to resolve numerically both the state and adjoint problems. Finally, the control equation is used as the reduced gradient in a gradientbased scheme to provide updates for the inversion variable/material property. The cycle is repeated, until convergence. The details are outlined below.

\subsection{The state problem - semi-discrete form}

Recall that the state problem is identical to the forward problem for a vibrating Timoshenko beam. Following standard lines, we multiply the governing PDEs of the state problem (2.2) and (2.3) by test functions $u(x)$ and 
$v(x)$, respectively, and integrate over the domain $(0, L)$; there results:

$$
\begin{aligned}
& \int_{0}^{L} u(x) \frac{\partial}{\partial x}\left\{G A K_{s}\left(\frac{\partial w}{\partial x}-\psi\right)\right\}-u(x) \rho A \frac{\partial^{2} w}{\partial t^{2}} \mathrm{~d} x \\
&=-\int_{0}^{L} u(x) q(x, t) \mathrm{d} x \\
& \int_{0}^{L} v(x) G A K_{s}\left(\frac{\partial w}{\partial x}-\psi\right)+v(x) \frac{\partial}{\partial x}(\left.E I \frac{\partial \psi}{\partial x}\right) \\
&-v(x) \rho I \frac{\partial^{2} \psi}{\partial t^{2}} \mathrm{~d} x=0 .
\end{aligned}
$$

Thus,

$$
\begin{gathered}
{\left[u G A K_{s}\left(\frac{\partial w}{\partial x}-\psi\right)\right]_{0}^{L}-\int_{0}^{L} G A K_{s} \frac{\partial w}{\partial x} \frac{\partial u}{\partial x} \mathrm{~d} x+\int_{0}^{L} G A K_{s} \psi \frac{\partial u}{\partial x} \mathrm{~d} x} \\
-\int_{0}^{L} u \rho A \frac{\partial^{2} w}{\partial t^{2}} \mathrm{~d} x=-\int_{0}^{L} u q \mathrm{~d} x \\
\int_{0}^{L} v G A K_{s} \frac{\partial w}{\partial x}-v G A K_{s} \psi \mathrm{d} x+\left[v E I \frac{\partial \psi}{\partial x}\right]_{0}^{L}-\int_{0}^{L} E I \frac{\partial v}{\partial x} \frac{\partial \psi}{\partial x} \mathrm{~d} x \\
-\int_{0}^{L} v \rho I \frac{\partial^{2} \psi}{\partial t^{2}} \mathrm{~d} x=0 .
\end{gathered}
$$

We then use boundary conditions (3.8) - (3.11) to arrive at the following weak forms. 


$$
\begin{gathered}
-\int_{0}^{L} G A K_{s} \frac{\partial w}{\partial x} \frac{\partial u}{\partial x} \mathrm{~d} x+\int_{0}^{L} G A K_{s} \psi \frac{\partial u}{\partial x} \mathrm{~d} x-\int_{0}^{L} u \rho A \frac{\partial^{2} w}{\partial t^{2}} \mathrm{~d} x \\
=-\int_{0}^{L} u q \mathrm{~d} x \\
\int_{0}^{L} v G A K_{s} \frac{\partial w}{\partial x}-v G A K_{s} \psi \mathrm{d} x-\int_{0}^{L} E I \frac{\partial v}{\partial x} \frac{\partial \psi}{\partial x} \mathrm{~d} x \\
-\int_{0}^{L} v \rho I \frac{\partial^{2} \psi}{\partial t^{2}} \mathrm{~d} x=0 .
\end{gathered}
$$

Next, the test $u, v$, and trial functions $w, \psi$ are approximated by:

$$
\begin{aligned}
w(x, t) & \simeq \phi(x)^{T} \mathbf{W}(t), \\
u(x) & \simeq \mathbf{u}^{T} \boldsymbol{\phi}(x), \\
\psi(x, t) & \simeq \mathbf{g}(x)^{T} \mathbf{\Psi}(t), \\
v(x) & \simeq \mathbf{v}^{T} \mathbf{g}(x),
\end{aligned}
$$

where $\mathbf{W}(t)$ and $\mathbf{\Psi}(t)$ are vectors of unknown nodal deflections and slopes, respectively, and $\phi(x)$ and $\mathbf{g}(x)$ are vectors of shape functions. For the latter, there are several choices proposed in the literature (Kapur [9];Thomas and Abbas [19];Reddy [14]). Among those, we favor consistent choices, whereby the order of the approximant for the deflections remains one order higher than that of the slopes, to avoid spurious energy modes, or the need for underintegration. To this end, we select standard Lagrange 4-noded cubic polynomials to approximate $w$ (and $u$ ), and Lagrange 3-noded quadratic polynomials to approximate $\psi$ (and $v$ ); we use linear interpolants $\mathbf{h}(x)$ for $E(x)$ (Fig. 4.1). Given these choices, the element mass and stiffness matrices are $7 \times 7$. 


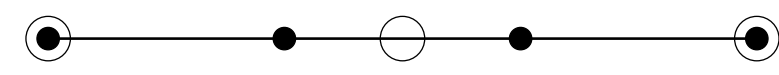

- Nodal deflections - w(x,t) (cubic)

Nodal slopes $-\psi(\mathrm{x}, \mathrm{t})$ (quadratic)

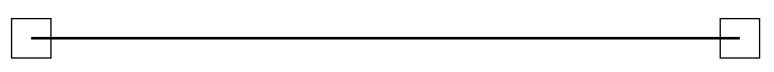

Nodal moduli - E(x) (linear)

Figure 4.1: Nodes associated with the element interpolants for the nodal deflections $w$, nodal slopes $\psi$, and nodal Young's moduli $E$

Introduction of (4.5)-(4.8) in the weak forms (4.3)-(4.4) yields the following semi-discrete system:

$$
\mathrm{M}_{\mathrm{st}} \ddot{\mathbf{d}}_{\mathrm{st}}+\mathbf{K}_{\mathrm{st}} \mathbf{d}_{\mathrm{st}}=\mathrm{Q}_{\mathrm{st}},
$$

where a dot denotes time-derivative of the subtended quantity, and,

$$
\begin{aligned}
& \mathbf{M}_{\mathrm{st}}=\left[\begin{array}{cc}
\int_{0}^{L} \rho A \boldsymbol{\phi} \boldsymbol{\phi}^{T} \mathrm{~d} x & \mathbf{0} \\
\mathbf{0} & \int_{0}^{L} \rho I \mathbf{g} \mathbf{g}^{T} \mathrm{~d} x
\end{array}\right], \\
& \mathbf{K}_{\mathrm{st}}=\left[\begin{array}{cc}
\int_{0}^{L} G A K_{s} \boldsymbol{\phi}^{\prime} \boldsymbol{\phi}^{\prime T} \mathrm{~d} x & \int_{0}^{L} G A K_{s} \boldsymbol{\phi}^{\prime} \mathbf{g}^{T} \mathrm{~d} x \\
\int_{0}^{L} G A K_{s} \mathbf{g} \boldsymbol{\phi}^{\prime T} \mathrm{~d} x & \int_{0}^{L}\left(G A K_{s} \mathbf{g} \mathbf{g}^{T}+E I \mathbf{g}^{\prime} \mathbf{g}^{T}\right) \mathrm{d} x
\end{array}\right], \\
& \mathbf{d}_{\mathrm{st}}=\left[\begin{array}{c}
\mathbf{W} \\
\mathbf{\Psi}
\end{array}\right], \\
& \mathbf{Q}_{\mathrm{st}}=\left[\begin{array}{c}
-\int_{0}^{L} \boldsymbol{\phi} q \mathrm{~d} x \\
\mathbf{0}
\end{array}\right] .
\end{aligned}
$$


In equations (4.9) to (4.13), $\mathbf{M}_{\mathrm{st}}$ and $\mathbf{K}_{\mathrm{st}}$ represent the mass and stiffness matrices respectively. $\mathbf{Q}_{\text {st }}$ is the load vector. $\mathbf{d}_{\text {st }}$ is the vector of unknown nodal values of $w$ and $\psi$.

\subsection{The adjoint problem - semi-discrete form}

As remarked in section 3.2.3, the governing operators for the state and adjoint problems are identical. Thus, the finite element model for the adjoint problem involves similar mathematical considerations as those for the state problem. $\lambda_{w}$ and $\lambda_{\psi}$ are treated as independent variables and the same test functions, $u(x)$ and $v(x)$, are used.

$$
\begin{aligned}
& \int_{0}^{L} u(x) \frac{\partial}{\partial x}\left\{G A K_{s}\left(\frac{\partial \lambda_{w}}{\partial x}-\lambda_{\psi}\right)\right\}- u(x) \rho A \frac{\partial^{2} \lambda_{w}}{\partial t^{2}} \mathrm{~d} x \\
&=-\int_{0}^{L} u(x) \theta(x, t) \mathrm{d} x \\
& \int_{0}^{L} v(x) G A K_{s}\left(\frac{\partial \lambda_{w}}{\partial x}-\lambda_{\psi}\right)+v(x) \frac{\partial}{\partial x}\left(E I \frac{\partial \lambda_{\psi}}{\partial x}\right) \\
&-v(x) \rho I \frac{\partial^{2} \lambda_{\psi}}{\partial t^{2}} \mathrm{~d} x=0
\end{aligned}
$$

where $\theta$ is the driver for the adjoint problem, defined as:

$$
\theta(x, t)=\sum_{i=1}^{N}\left[w^{m}(x, t)-w(x, t)\right] \delta\left(x-x_{i}\right) .
$$

We then use integration by parts and boundary conditions (3.45) - (3.48) to get the following weak forms. 


$$
\begin{array}{rl}
-\int_{0}^{L} G A K_{s} \frac{\partial \lambda_{w}}{\partial x} \frac{\partial u}{\partial x} \mathrm{~d} x+\int_{0}^{L} G A K_{s} \lambda_{\psi} & \frac{\partial u}{\partial x} \mathrm{~d} x-\int_{0}^{L} u \rho A \frac{\partial^{2} \lambda_{w}}{\partial t^{2}} \mathrm{~d} x \\
& =-\int_{0}^{L} u \theta(x, t) \mathrm{d} x \\
\int_{0}^{L} v G A K_{s} \frac{\partial \lambda_{w}}{\partial x}-v G A K_{s} \lambda_{\psi} \mathrm{d} x-\int_{0}^{L} & E I \frac{\partial v}{\partial x} \frac{\partial \lambda_{\psi}}{\partial x} \mathrm{~d} x \\
& -\int_{0}^{L} v \rho I \frac{\partial^{2} \lambda_{\psi}}{\partial t^{2}} \mathrm{~d} x=0
\end{array}
$$

Again, we select Lagrange cubic polynomials to approximate $\lambda_{w}$, and Lagrange quadratic polynomials to approximate $\lambda_{\psi}$. The following approximations for trial and test functions $-\left\{\lambda_{w}(x, t), u(x), \lambda_{\psi}(x, t), v(x)\right\}$ are obtained:

$$
\begin{aligned}
\lambda_{w}(x, t) & \simeq \boldsymbol{\phi}(x)^{T} \boldsymbol{\lambda}_{\boldsymbol{w}}(t), \\
u(x) & \simeq \mathbf{u}^{T} \boldsymbol{\phi}(x), \\
\lambda_{\psi}(x, t) & \simeq \mathrm{g}(x)^{T} \boldsymbol{\lambda}_{\psi}(t), \\
v(x) & \simeq \mathbf{v}^{T} \mathbf{g}(x) .
\end{aligned}
$$

Introduction of (4.18) - (4.21) in the weak forms (4.16), (4.17) yields the following semi-discrete system:

$$
\mathbf{M}_{\mathrm{adj}} \ddot{\mathbf{d}}_{\mathrm{adj}}+\mathbf{K}_{\mathrm{adj}} \mathbf{d}_{\mathrm{adj}}=\mathbf{Q}_{\mathrm{adj}}
$$

where a dot denotes time-derivative of the subtended quantity, and, 


$$
\begin{aligned}
\mathbf{M}_{\mathrm{adj}}=\mathbf{M}_{\mathrm{st}} & , \quad \mathbf{K}_{\mathrm{adj}}=\mathbf{K}_{\mathrm{st}}, \\
\mathbf{d}_{\mathrm{adj}} & =\left[\begin{array}{c}
\boldsymbol{\lambda}_{\boldsymbol{w}} \\
\boldsymbol{\lambda}_{\boldsymbol{\psi}}
\end{array}\right], \\
\mathbf{Q}_{\mathrm{adj}} & =\left[\begin{array}{c}
-\int_{0}^{L} \boldsymbol{\phi} \theta \mathrm{d} x \\
\mathbf{0}
\end{array}\right] .
\end{aligned}
$$

Due to (4.23), the computational cost associated with matrix assembly is reduced considerably, since the matrices need to be assembled only once during each inversion iteration for both the state and adjoint problems. The time integration procedure for the state and adjoint problems is, however, slightly different, since the state problem is an initial value problem, whereas the adjoint problem is a final value problem. We address these schemes next.

\subsection{State and adjoint time integration}

\subsubsection{Time integration for the state problem}

We use standard Newmark average acceleration schemes for both the state and adjoint problems. Denoting with a superscript $n$ the subtended quantity's value at the $n$-th time-step, the resulting scheme for the state problem can be summarized as:

$$
\mathbf{M}_{\mathrm{st}}^{\mathrm{eff}} \ddot{\mathbf{d}}_{\mathrm{st}}^{n+1}=\mathbf{Q}_{\mathrm{st}}^{\mathrm{eff}},
$$


where,

$$
\begin{aligned}
\mathbf{M}_{\mathrm{st}}^{\mathrm{eff}} & =\mathbf{M}_{\mathrm{st}}+\frac{1}{4} \Delta t^{2} \mathbf{K}_{\mathrm{st}}, \\
\mathbf{Q}_{\mathrm{st}}^{\mathrm{eff}} & =\mathbf{Q}_{\mathrm{st}}^{n+1}-\mathbf{K}_{\mathrm{st}} \mathbf{d}_{\mathrm{st}}^{n}-\Delta t \mathbf{K}_{\mathrm{st}} \dot{\mathbf{d}}_{\mathrm{st}}^{n}-\frac{1}{4} \Delta t^{2} \mathbf{K}_{\mathrm{st}} \ddot{\mathbf{d}}_{\mathrm{st}}^{n},
\end{aligned}
$$

and $\Delta t$ is the time-step. To initiate the process, we use silent initial conditions and the following equation:

$$
\mathbf{M}_{\mathrm{st}} \ddot{\mathbf{d}}_{\mathrm{st}}^{0}=\mathrm{Q}_{\mathrm{st}}^{0}-\mathbf{K}_{\mathrm{st}} \mathbf{d}_{\mathrm{st}}^{0}
$$

Upon recovering of the accelerations $\ddot{\mathbf{d}}_{\mathrm{st}}^{n+1}$, the velocities and the displacements can be obtained using:

$$
\begin{aligned}
\dot{\mathbf{d}}_{\mathrm{st}}^{n+1} & =\dot{\mathbf{d}}_{\mathrm{st}}^{n}+\frac{1}{2} \Delta t\left[\ddot{\mathbf{d}}_{\mathrm{st}}^{n}+\ddot{\mathbf{d}}_{\mathrm{st}}^{n+1}\right], \\
\mathbf{d}_{\mathrm{st}}^{n+1} & =\mathbf{d}_{\mathrm{st}}^{n}+\Delta t \dot{\mathbf{d}}_{\mathrm{st}}^{n}+\frac{1}{4} \Delta t^{2}\left[\ddot{\mathbf{d}}_{\mathrm{st}}^{n}+\ddot{\mathbf{d}}_{\mathrm{st}}^{n+1}\right] .
\end{aligned}
$$

\subsubsection{Time integration for the adjoint problem}

By contrast to the state problem, the adjoint problem is initiated using the final time conditions at $t=T$, to obtain the final time adjoint "accelerations," as:

$$
\mathbf{M}_{\mathrm{adj}} \ddot{\mathbf{d}}_{\mathrm{adj}}^{\mathrm{T}}=\mathbf{Q}_{\mathrm{adj}}^{\mathrm{T}}-\mathbf{K}_{\mathrm{adj}} \mathbf{d}_{\mathrm{adj}}^{\mathrm{T}} \text {. }
$$


To resolve the accelerations at subsequent time-steps, we traverse the time-line in the negative direction, and adjust the Newmark scheme accordingly:

$$
\mathbf{M}_{\text {adj }}^{\text {eff }} \ddot{\mathbf{d}_{s t}^{n-1}}=\mathbf{Q}_{s t}^{\mathrm{eff}}
$$

where,

$$
\begin{aligned}
\mathbf{M}_{\mathrm{adj}}^{\mathrm{eff}}= & \mathbf{M}_{\mathrm{adj}}+\frac{\Delta t^{2}}{4} \mathbf{K}_{\mathrm{adj}}=\mathbf{M}_{\mathrm{st}}^{\mathrm{eff}}, \\
\mathbf{Q}_{\mathrm{adj}}^{\mathrm{eff}}= & \mathbf{Q}_{\mathrm{adj}}^{n-1}-\mathbf{K}_{\mathrm{adj}} \mathbf{d}_{\mathrm{adj}}^{n}+\Delta t \mathbf{K}_{\mathrm{adj}} \dot{\mathbf{d}}_{\mathrm{adj}}^{n} \\
& -\frac{1}{4} \Delta t^{2} \mathbf{K}_{\mathrm{adj}} \ddot{\mathbf{d}}_{\mathrm{adj}}^{n} .
\end{aligned}
$$

The values of $\dot{\mathbf{d}}_{\text {adj }}^{n-1}$ and $\mathbf{d}_{\text {adj }}^{n-1}$ can then be computed using:

$$
\begin{aligned}
\dot{\mathbf{d}}_{\mathrm{adj}}^{n-1} & =\dot{\mathbf{d}}_{\mathrm{adj}}^{n}-\frac{1}{2} \Delta t\left[\ddot{\mathbf{d}}_{\mathrm{adj}}^{n-1}+\ddot{\mathbf{d}}_{\mathrm{adj}}^{n}\right] \\
\mathbf{d}_{\mathrm{adj}}^{n-1} & =\mathbf{d}_{\mathrm{adj}}^{n}-\Delta t \dot{\mathbf{d}}_{\mathrm{adj}}^{n}+\frac{1}{4} \Delta t^{2}\left[\ddot{\mathbf{d}}_{\mathrm{adj}}^{n-1}+\ddot{\mathbf{d}}_{\mathrm{adj}}^{n}\right] .
\end{aligned}
$$

\subsection{Inversion parameter updates}

The reconstruction of the distributed Young's modulus $E(x)$ begins with an initial guess. Then, the state variables $w(x, t), \psi(x, t)$ satisfying equations (3.6)-(3.14) are computed. Next, the adjoint variables $\lambda_{w}(x, t), \lambda_{\psi}(x, t)$, satisfying equations (3.43) - (3.52) are obtained. We, then, seek fulfillment of the control problem (3.54) or (3.55), using the values of the state and adjoint 
variables, as well as the current profile of the inversion variable $E(x)$. The control problem is readily satisfied if the current profile of the inversion variable is the true profile. This is not the case, in general, and an iterative procedure is required to recover the profile: such a procedure can be readily devised by noticing that the non-vanishing left-hand-side of the control equation is the reduced gradient of the Lagrangian functional $\left(\nabla_{E} \mathcal{L}\right)$. Furthermore, the reduced gradient is equal to the gradient of the objective functional $\left(\nabla_{E} \mathcal{F}\right)$, since the side constraints vanish on account of satisfaction of the state problem. Thus: Reduced gradient - TN regularization

$$
\begin{aligned}
\nabla_{E} \mathcal{L}= & -R_{E} \frac{d^{2} E}{d x^{2}} \\
& -\int_{0}^{T}\left[\frac{A K_{s}}{2(1+\nu)}\left(\frac{\partial w}{\partial x}-\psi\right)\left(\frac{\partial \lambda_{w}}{\partial x}-\lambda_{\psi}\right)\right. \\
& \left.+I \frac{\partial \psi}{\partial x} \frac{\partial \lambda_{\psi}}{\partial x}\right] \mathrm{d} t
\end{aligned}
$$

subject to $\left(\frac{d E}{d x}\right)_{x=0}=0$ and $\left(\frac{d E}{d x}\right)_{x=L}=0$.

Reduced gradient - TV regularization

$$
\begin{aligned}
\nabla_{E} \mathcal{L}= & -R_{E} \epsilon \frac{d^{2} E}{d x^{2}}\left[\left(\frac{d E}{d x}\right)^{2}+\epsilon\right]^{-\frac{3}{2}} \\
- & \int_{0}^{T}\left[\frac{A K_{s}}{2(1+\nu)}\left(\frac{\partial w}{\partial x}-\psi\right)\left(\frac{\partial \lambda_{w}}{\partial x}-\lambda_{\psi}\right)\right. \\
+ & \left.I \frac{\partial \psi}{\partial x} \frac{\partial \lambda_{\psi}}{\partial x}\right] \mathrm{d} t,
\end{aligned}
$$

subject also to $\left(\frac{d E}{d x}\right)_{x=0}=0$ and $\left(\frac{d E}{d x}\right)_{x=L}=0$.

From this point on, there are three options for handling the inversion variable updates: one could treat (4.38) or (4.39) as search directions in a gradient- 
based scheme, or seek to enforce the control problem (3.54) or (3.55) in a weak sense, using the variation $\delta E$ as the weight function. We discuss all three schemes and highlight the relative merits; we focus first on the gradient-based scheme using a conjugate-gradient method.

\subsubsection{Gradient-based scheme for inversion variable updates}

Let $\mathbf{g}_{k}$ be the (discretized) reduced gradient (4.38) or (4.39) at the $k$-th inversion iteration, i.e.,

$$
\mathbf{g}_{k}=\left(\nabla_{E} \mathcal{L}\right)_{k}
$$

Moreover, let the vector $\mathbf{E}^{(k)}$ denote the nodal values of the inversion variable $E$ at the $k$-th iteration. We compute the updated vector $\mathbf{E}^{(k+1)}$ of the inversion variable at the next iteration as:

$$
\mathbf{E}^{(k+1)}=\mathbf{E}^{(k)}+\alpha \mathbf{d}_{k},
$$

where $\mathbf{d}_{k}$ is the search direction, and $\alpha$ is the step length. The search direction $\mathbf{d}_{k}$ can be obtained as:

$$
\mathbf{d}_{k}= \begin{cases}-\mathbf{g}_{k} & \text { if } k=0 \\ -\mathbf{g}_{k}+\frac{\mathbf{g}_{k} \cdot \mathbf{g}_{k}}{\mathbf{g}_{k-1} \cdot \mathbf{g}_{k-1}} \mathbf{d}_{k-1} & \text { if } k \geq 1\end{cases}
$$

Thus, with the exception of the first iteration, the search direction is updated at every inversion iteration based on the reduced gradient of the previous iteration. However, in practice, due to round-off errors that lead to the progressive 
contamination of the search direction, $\mathbf{d}_{k}$ is reset every $m$ iterations (we used $m=10)$; thus, the scheme is modified to read:

$$
\mathbf{d}_{k}=\left\{\begin{array}{lc}
-\mathbf{g}_{k} & \text { if } \bmod (k, m)=0 \\
-\mathbf{g}_{k}+\frac{\mathbf{g}_{k} \cdot \mathbf{g}_{k}}{\mathbf{g}_{k-1} \cdot \mathbf{g}_{k-1}} \mathbf{d}_{k-1} & \text { otherwise }
\end{array}\right.
$$

Once the update $\mathbf{E}^{(k+1)}$ is obtained, we evaluate the misfit functional $\mathcal{J}$ and compare it against a preset tolerance. If the misfit is less than the tolerance, the inversion process is terminated, and $\mathbf{E}^{(k+1)}$ is regarded as the stationary solution. Otherwise, the outlined process is repeated. Lastly, to determine appropriate values for the step length $\alpha$ in (4.41), an inexact line search with backtracking is employed, subject to the Armijo condition. The condition requires that $\alpha$ produce a sufficient decrease in the misfit functional, that is:

$$
\mathcal{J}\left(\mathbf{E}^{(k)}+\alpha \mathbf{d}_{k}\right) \leq \mathcal{J}\left(\mathbf{E}^{(k)}\right)+\mu \alpha \mathbf{g}_{k} \cdot \mathbf{d}_{k},
$$

for which we used $\mu=10^{-12}$. In order to obtain an $\alpha$ satisfying (4.44), the backtracking approach is utilized (Nocedal and Wright [12]). The process is initialized with a suitable step length $\alpha=\alpha_{o}$. Then, the step length $\alpha$ is reduced by letting $\alpha \leftarrow \rho \alpha$ for $\rho<1$ until the Armijo condition is satisfied ( $\rho=0.5$ was used throughout). The entire inversion process is summarized in Table 4.1.

Remarks

- We note that the reduced gradients (4.38) or (4.39) require that the first derivatives of the inversion variable vanish at domain ends $((d E / d x)=0$ 
Algorithm 1 Inversion algorithm using a reduced space method

1: Choose $\alpha_{0}, \rho, \mu$ and $R_{E}$; Set $\alpha=\alpha_{0}$

2: $\quad$ Set $\mathrm{k}=0$ and convergence tolerance tol

3: Set initial guess of the inversion variable $\mathbf{c}_{k}$

4: $\quad$ Set $\mathcal{J}=t o l+1$

5: $\quad$ while $(\mathcal{J}>t o l)$ do

6: $\quad$ Solve the state problem (3.6),(3.7), (3.8) - (3.14) to get $w$ and $\psi$

7: $\quad$ Solve the adjoint problem $(3.43),(3.44),(3.45)-(3.52)$ to get $\lambda_{w}$ and $\lambda_{\psi}$

8: $\quad$ Calculate the discrete form of reduced gradient $\mathbf{g}_{k}=\left(\nabla_{E} \mathcal{L}\right)_{k}$

9: $\quad$ Compute the search direction $\mathbf{d}_{k}$ using (4.43)

10: $\quad$ while $\left[\mathcal{F}\left(\mathbf{c}_{k}+\alpha \mathbf{d}_{k}\right) \leq \mathcal{F}\left(\mathbf{c}_{k}\right)+\mu \alpha \mathbf{g}_{k} \cdot \mathbf{d}_{k}\right]$ do

11: $\quad \alpha \leftarrow \rho \alpha$

12: $\quad$ end while

13: $\quad$ Update the material property vector by $\mathbf{c}_{k+1}=\mathbf{c}_{k}+\alpha \mathbf{d}_{k}$

14: $\quad k=k+1$

15: end while

Table 4.1: Inversion algorithm (Kang and Kallivokas [8]) 
at $x=0, L)$. We explicitly impose these requirements by forcing a constant value of $E$ over the extreme elements of the domain: this action realizes, in a finite-difference sense, the vanishing of the derivatives.

- We also remark that the direct use of (4.38) or (4.39) requires the computation of the second spatial derivative of the inversion variable $E$ at element ends. Since $E$ is approximated using, in general, $C^{0}$ interpolants (linear herein), its second derivative is a Dirac function at nodal locations. We use a central finite-difference scheme to approximate the second derivative, effectively smoothening numerically a Dirac function. Though the finite difference approximation is inconsistent with the underlying assumptions, we have not observed an impact on the convergence rate, or the quality of the results. Alternatively, one could use the scheme we describe next, which avoids the explicit computation of the second derivatives, while also avoiding the need for the explicit imposition of the vanishing of the first derivatives at the domain ends.

\subsubsection{Weak-form-based scheme for inversion variable updates}

We return to the control problem expression (3.54) (or (3.55)), and use $\delta E$ as a weight function, approximated by:

$$
\delta E=\mathbf{z}^{T} \mathbf{h}(x),
$$

where $\mathbf{z}^{T}$ are arbitrary nodal quantities, and $\mathbf{h}(x)$ is the vector of linear approximants (recall $E(x)$ is similarly approximated by $\mathbf{h}(x)$ ). After integration 
by parts, there results:

$$
\begin{gathered}
\int_{0}^{L} \frac{d \mathbf{h}(x)}{d x} \frac{d \mathbf{h}(x)^{T}}{d x} \mathrm{~d} x \mathbf{E}- \\
\frac{1}{R_{E}} \int_{0}^{T} \int_{0}^{L} \mathbf{h}(x)\left[\frac{A K_{s}}{2(1+\nu)}\left(\frac{\partial w}{\partial x}-\psi\right)\left(\frac{\partial \lambda_{w}}{\partial x}-\lambda_{\psi}\right)\right. \\
\left.+I \frac{\partial \psi}{\partial x} \frac{\partial \lambda_{\psi}}{\partial x}\right] \mathrm{d} x \mathrm{~d} t=0
\end{gathered}
$$

where $\mathbf{E}$ above is the vector of nodal $E(x)$ values. Equation (4.46) can be solved for the nodal values $\mathbf{E}$ of the inversion variable, without need to resort to a gradient-based scheme. The right-hand-side is readily computable at each inversion iteration, following the solution of the state and adjoint problems. The left-hand-side of (4.46) requires the computation of a "stiffness-like" matrix only once for all inversion iterations. The matrix, however, is singular with rank deficiency of one, due to the pure Neumann character of the problem: we recall that the only conditions imposed on $E(x)$ were the two Neumann end conditions $\left(\frac{d E}{d x}\right)_{x=0}$ and $\left(\frac{d E}{d x}\right)_{x=L}$. Thus, to overcome the singularity, the value of $E$ must be a priori fixed at one node of the discretization. This is, in effect, the only disadvantage of this second scheme for computing material updates.

Alternatively, (4.46) too can be used as the reduced gradient, in its weak form, to drive the gradient-based scheme. Of the three alternatives, the results presented herein were obtained using the first scheme. 


\subsection{Regularization factor continuation}

The choice of the regularization factor is, overall, critical to the successful recovery of the true material profile. Very small values of the regularization factor will not alleviate solution multiplicity, while large values may hinder reconstruction of the target profile. Here, we use a continuation scheme, in which the regularization factor for every inversion iteration is chosen based on values of the variables in the control equation (Kang and Kallivokas [8]). Thus,

$$
R_{E}=0.5 \frac{\left|\nabla_{E} \mathcal{F}_{m}\right|}{\left|\nabla_{E} \mathcal{F}_{r}\right|}
$$

where,

$$
\begin{aligned}
\nabla_{E} \mathcal{F}_{m}= & \int_{0}^{T}\left[\frac{A K_{s}}{2(1+\nu)}\left(\frac{\partial w}{\partial x}-\psi\right)\left(\frac{\partial \lambda_{w}}{\partial x}-\lambda_{\psi}\right)\right. \\
& \left.+I \frac{\partial \psi}{\partial x} \frac{\partial \lambda_{\psi}}{\partial x}\right] \mathrm{d} t, \\
\nabla_{E} \mathcal{F}_{r}= & \frac{d^{2} E}{d x^{2}} .
\end{aligned}
$$

The approach maintains a balance between the misfit part and the regularization part of the control equation leading to an improved estimate of $R_{E}$ over constant choices. Notice that in the initial part of the inversion, when the misfit is large, the regularization factor chosen by using equation (4.47) will be similarly large, and will thus assist in reducing the feasibility space of possible solutions. Once the misfit reduces sufficiently, a smaller regularization factor 
will present the optimizer with more flexibility in attaining the true profile (Kang and Kallivokas [8]). 


\section{Chapter 5}

\section{Numerical results}

We use the inversion methodology outlined in the previous chapters to first reconstruct material profiles of single beams. Extensions to frames are discussed in chapter 6 . We discuss the reconstruction of smoothly-varying profiles, which are typically easier to obtain using TN regularization, and the reconstruction of sharply-varying profiles, typically indicative of abrupt modulus changes, for which TV regularization is better suited. We also report on profiles characterized by localized damage extending over a fraction of the total component length. We experiment with distributed loads, which in practice will be more difficult to generate for condition assessment purposes, but report also quite satisfactory results with point loads, which are fairly easy to generate on existing structures. In all of the reported results, the measured responses have been generated synthetically, using a different discrete system than the one we use for inversion purposes to avoid biasing the inversion process.

We use a simply-supported beam to conduct the experiments, with nominal values for the various beam parameters. Specifically, we used: 


$$
\begin{aligned}
L & =1.0, \\
A(x) & =1.0, \\
I(x) & =\frac{1.0}{12.0}, \\
\rho(x) & =1.0, \\
K_{s} & =\frac{5}{6}, \\
q(x, t) & =\beta(x) \tau(t),
\end{aligned}
$$

where $\beta(x)$ and $\tau(t)$ denote the spatial and temporal dependence of the applied probing excitation $q(x, t)$. We consider two cases for $\beta$, a uniform load $\beta(x)=A_{o}$, and a point load $-\beta(x)=A_{o} \delta\left(x-x_{0}\right)$ applied at point $x=x_{0}$. Throughout all cases, a Gaussian time signal is used for $\tau(t)$ :

$$
\tau(t)=\exp \left[\frac{-\left(t-B_{o}\right)^{2}}{C_{o}}\right]
$$

where $A_{o}, B_{o}, C_{o}$ are user-defined parameters: the value of $C_{o}$ controls the frequency content of the time signal. The signal parameters, component geometry, load and sensor locations, are all shown in the figures that follow.

To start the process, the beam is subjected to the excitation $q(x, t)$ and the time history of the deflections is measured at the $N$ sensor locations. Generally, 3 or 4 sensors are used per member. In the next step, the time histories at the sensor locations are fed, via the misfit, as input to the inversion algorithm outlined earlier. The Young's modulus profile $E(x)$ corresponding 
to the stationary point of the augmented Lagrangian is recovered as output. Table 5.1 summarizes the data used for all the examples we report herein: as could be seen from the table, we used approximately 12-20 points per wavelength (using the smallest wavelength to drive the element size). For the cases exhibiting localized damage we remark that structural flaws were detected using excitations with shortest wavelength ranging from a fraction (Fig. 6.6) to about 4 wavelengths (Fig. 5.7) of the structural flaw's width.

\begin{tabular}{|c|c|c|c|c|c|}
\hline Example & $\lambda$ & $h_{e}$ & $\Delta t$ & $T$ & Iterations \\
\hline Fig. 5.1 & 0.0855 & 0.0200 & 0.010 & 4 & 2752 \\
\hline Fig. 5.2 & 0.0684 & 0.0125 & 0.008 & 4 & 2454 \\
\hline Fig. 5.3 & 0.0855 & 0.0100 & 0.010 & 4 & 11466 \\
\hline Fig. 5.4 & 0.0513 & 0.0100 & 0.006 & 4.2 & 4646 \\
\hline Fig. 5.5 & 0.0684 & 0.0200 & 0.008 & 4 & 8772 \\
\hline Fig. 5.6 & 0.0205 & 0.0050 & 0.002 & 3.6 & 8275 \\
\hline Fig. 5.7 & 0.0513 & 0.0100 & 0.006 & 4.2 & 2991 \\
\hline Fig. 6.2 & 0.1710 & 0.0125 & 0.020 & 10 & 4247 \\
\hline Fig. 6.3 & 0.0855 & 0.0100 & 0.008 & 13.2 & 1344 \\
\hline Fig. 6.4 & 0.0205 & 0.0050 & 0.004 & 6.6 & 1410 \\
\hline Fig. 6.5 & 0.0789 & 0.0067 & 0.008 & 6 & 990 \\
\hline
\end{tabular}

Table 5.1: Example characteristics: smallest wavelength $\lambda$, element size $h_{e}$, time-step $\Delta t$, total observation time $T$, number of iterations

Figures 5.1 and 5.2 show the results for smoothly varying profiles, which were reconstructed using 4 sensors, and a uniform and point load, respectively. Notice that in both cases the initial guess was a uniform profile that is not related to any prior undamaged component state. The recovered profiles match the targets quite well. Sharp profiles are similarly well reconstructed, as evidenced by the results shown in figures 5.3 and 5.4 pertaining to an abrupt $50 \%$ 
change in the modulus over the central $20 \%$ portion of the beam, which could represent a region experiencing diffuse cracking. Of similar quality are the reconstructed results depicted in figures 5.5 and 5.6. The former pertains to a staircase-type profile, while the latter depicts localized damage of different intensity in two neighboring sections. Lastly, Fig. 5.7 depicts the reconstructed profile obtained when there is $10 \%$ Gaussian noise infused in the synthetic data. Despite the noise, the profile is still fairly well reconstructed. 


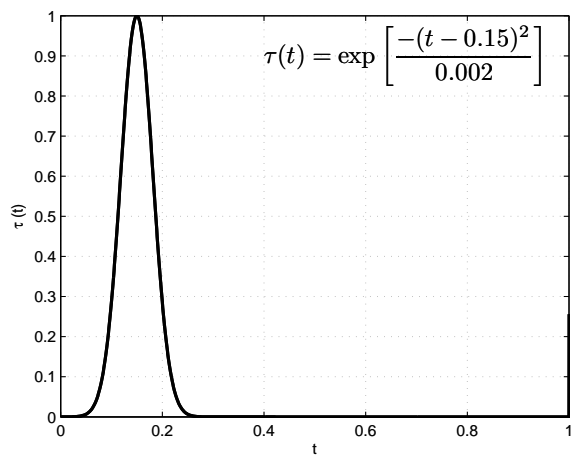

(a) Time signal $\tau(t)$

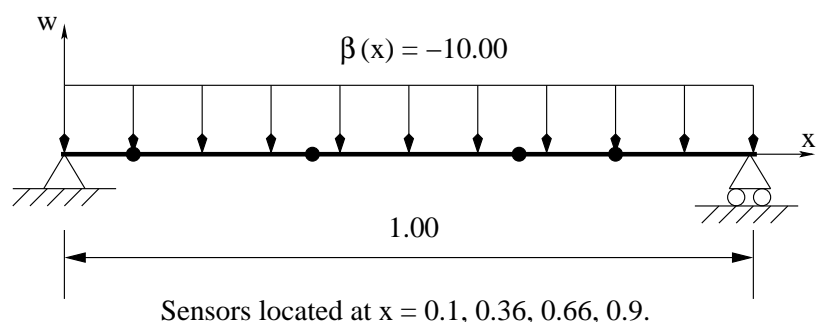

(b) Geometry, uniform load, and sensor locations

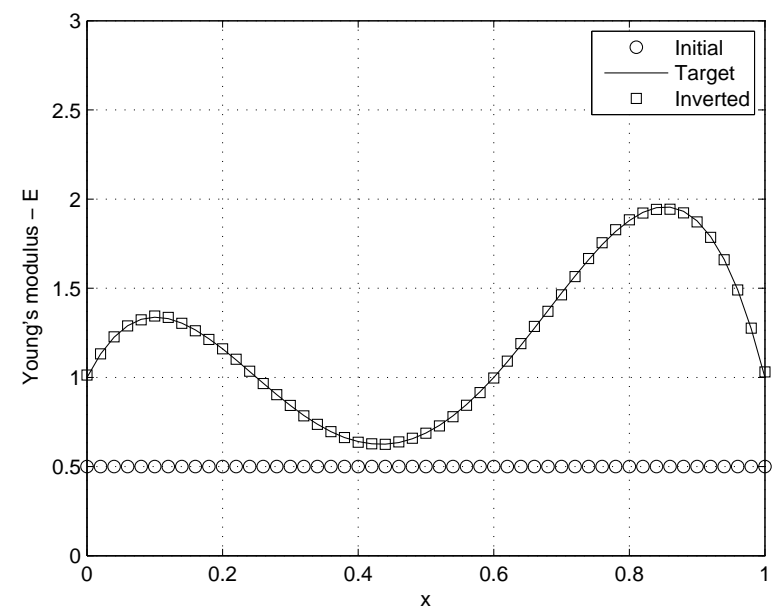

(c) Initial, target, and converged profile

Figure 5.1: Reconstruction of a smooth target profile for a simply-supported beam: excitation, uniform load, sensor distribution, TN regularization 


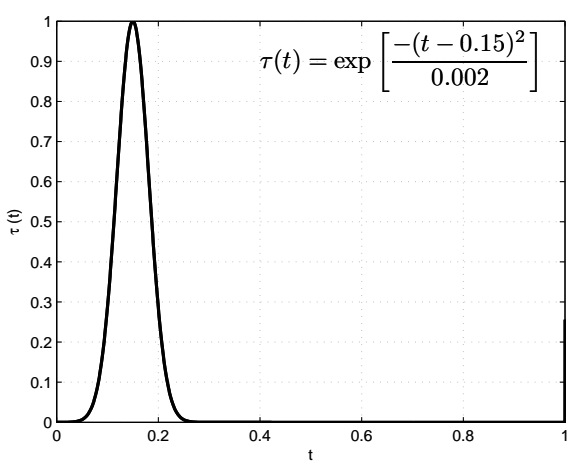

(a) Time signal $\tau(t)$

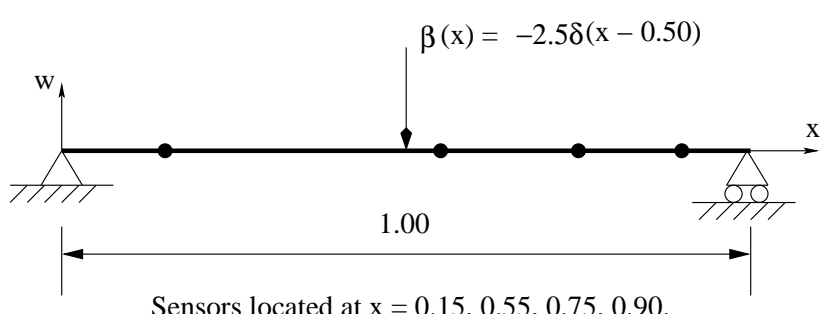

(b) Geometry, point load, and sensor locations

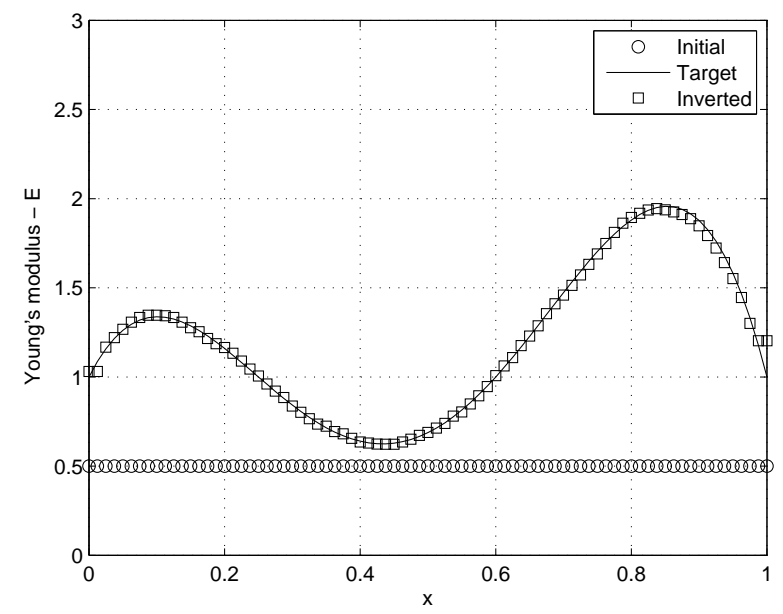

(c) Initial, target, and converged profile

Figure 5.2: Reconstruction of a smooth target profile for a simply-supported beam: excitation, point load location, sensor distribution, TN regularization 


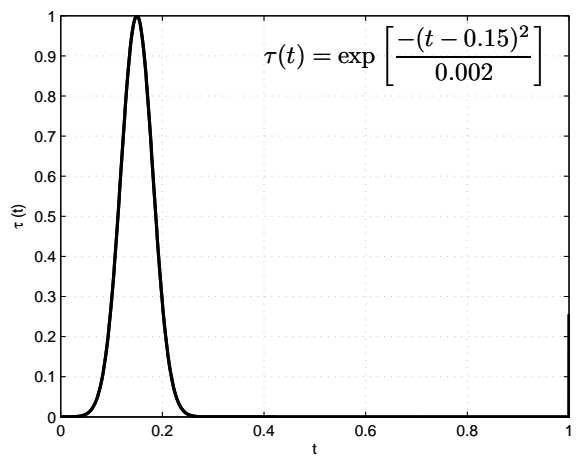

(a) Time signal $\tau(t)$

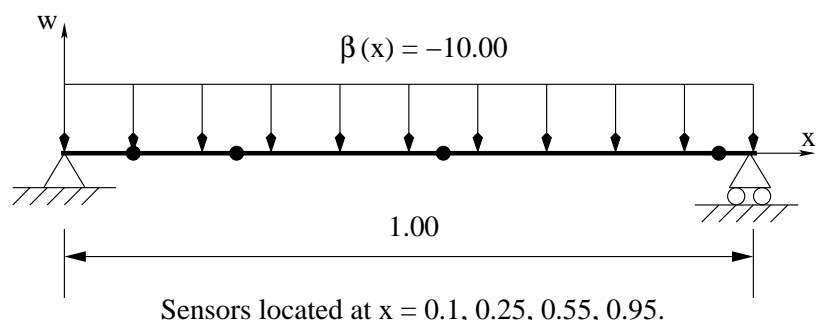

(b) Geometry, uniform load, and sensor locations

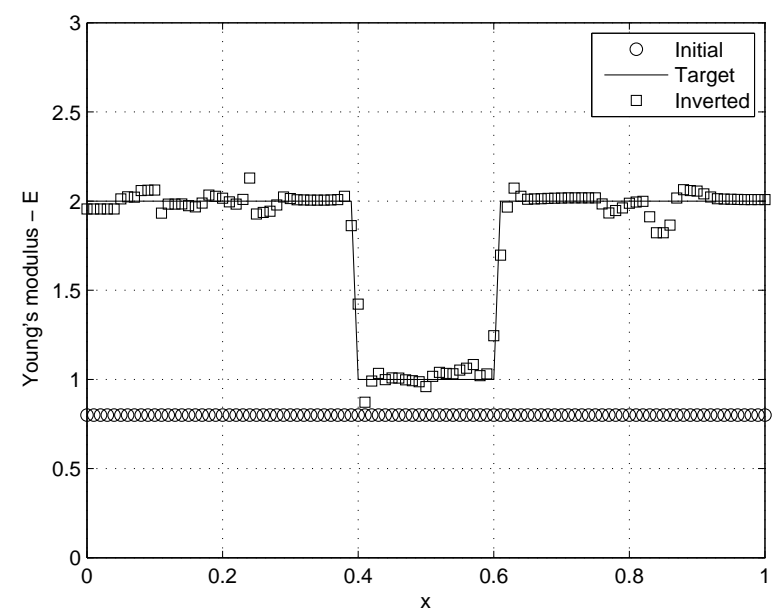

(c)

Figure 5.3: Reconstruction of a sharp target profile for a simply-supported beam: excitation, uniform load, sensor distribution, TV regularization 


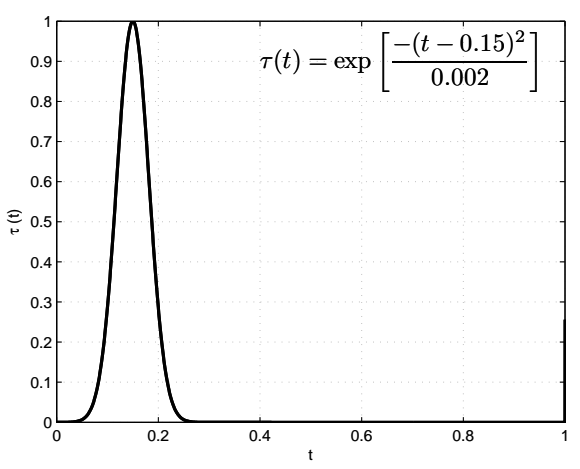

(a) Time signal $\tau(t)$

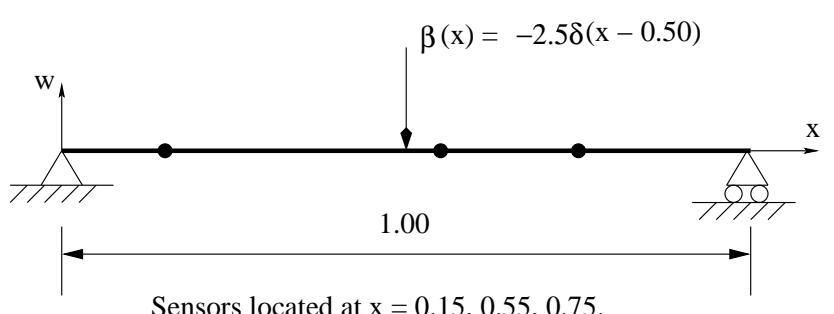

(b) Geometry, point load, and sensor locations

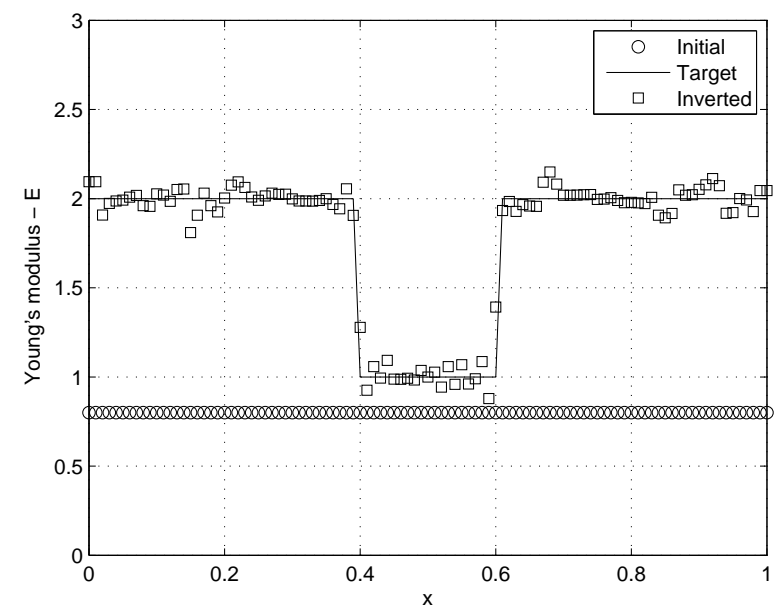

(c) Initial, target, and converged profile

Figure 5.4: Reconstruction of a sharp target profile for a simply-supported beam: excitation, point load location, sensor distribution, TV regularization 


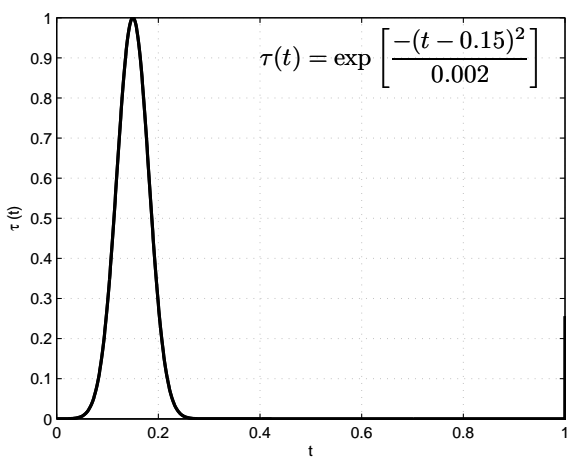

(a) Time signal $\tau(t)$

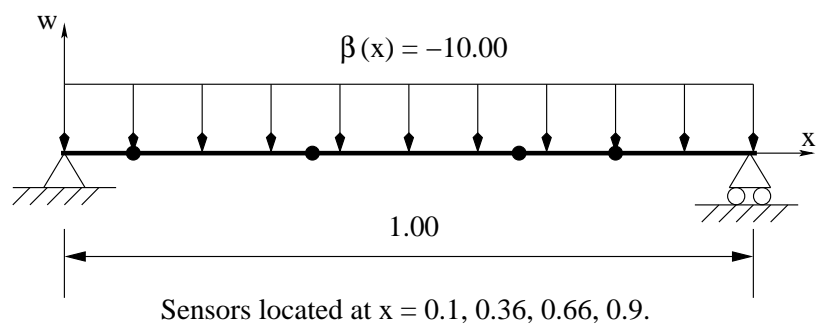

(b) Geometry, uniform load, and sensor locations

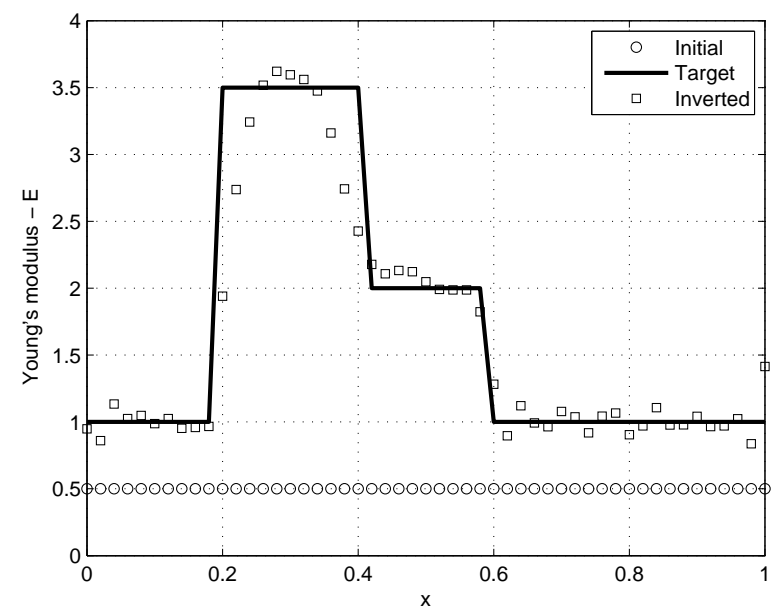

(c) Initial, target, and converged profile

Figure 5.5: Reconstruction of a multiply-sharp target profile for a simplysupported beam: excitation, uniform load, sensor distribution, TV regularization 


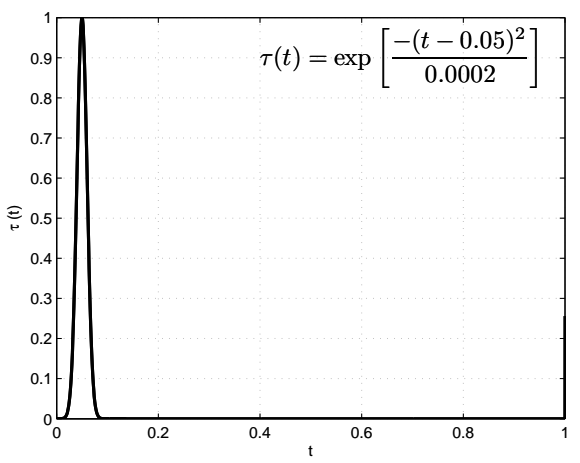

(a) Time signal $\tau(t)$

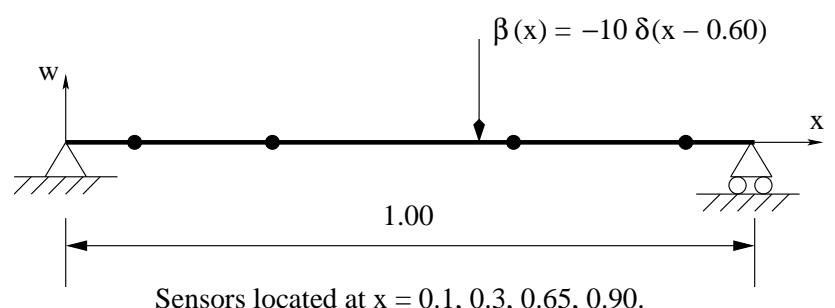

(b) Geometry, uniform load, and sensor locations

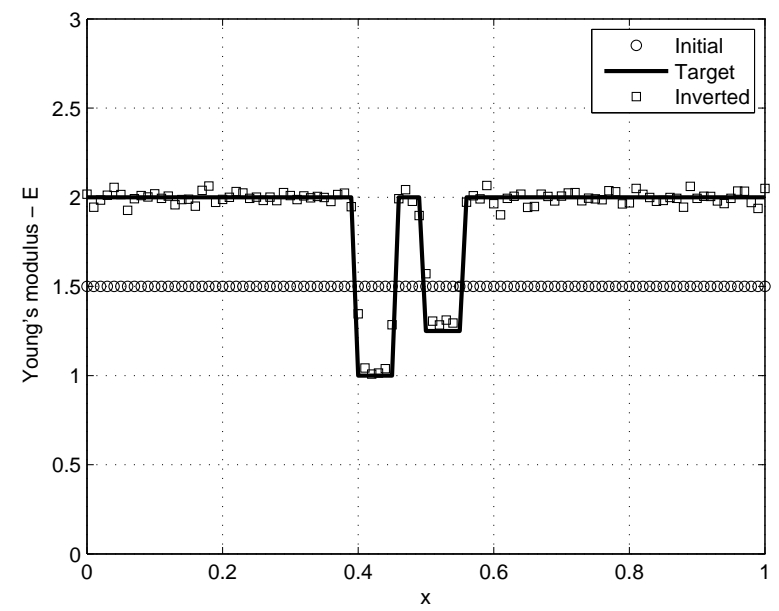

(c) Initial, target, and converged profile

Figure 5.6: Reconstruction of localized damage for a simply-supported beam: excitation, point load location, sensor distribution, TV regularization 


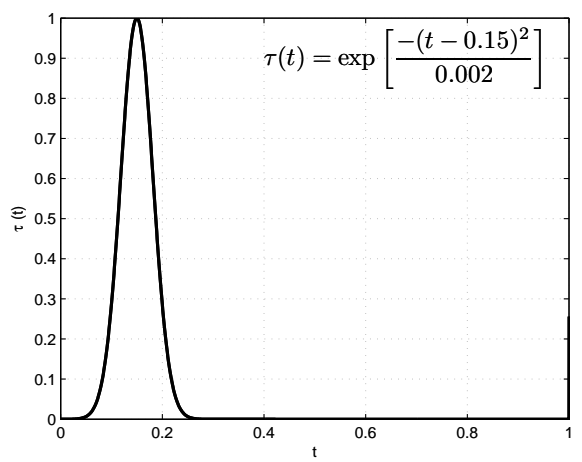

(a) Time signal $\tau(t)$

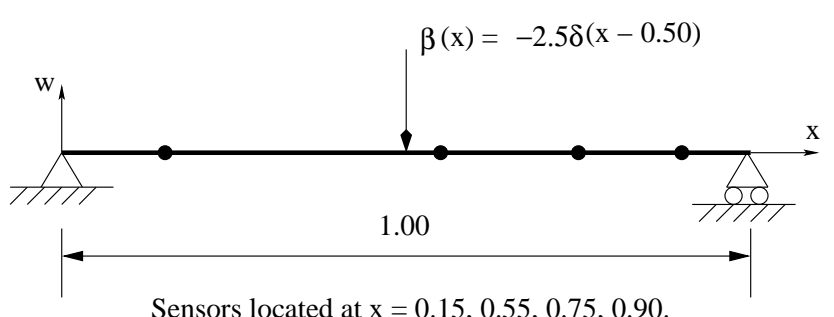

(b) Geometry, point load, and sensor locations

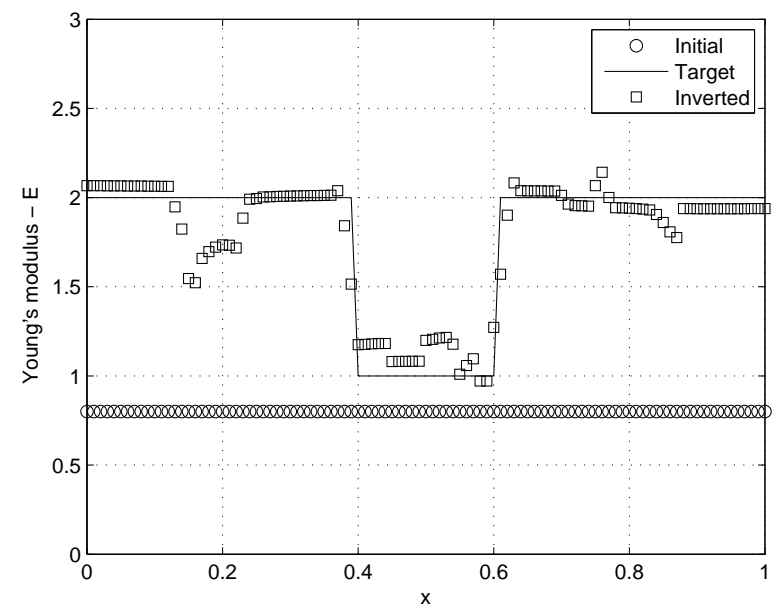

(c) Initial, target, and converged profile

Figure 5.7: Reconstruction of a sharp target profile for a simply-supported beam with $10 \%$ Gaussian noise in the data: excitation, point load location, sensor distribution, TV regularization 


\section{Chapter 6}

\section{Extensions}

The outlined process could be generalized to accommodate damage detection and profile reconstruction in frames. In addition, the inversion process could be cast in terms of the second moment of inertia, instead of Young's modulus, if a geometric measure of damage is desired. Both extensions are addressed below.

\subsection{Material profile reconstruction for frames}

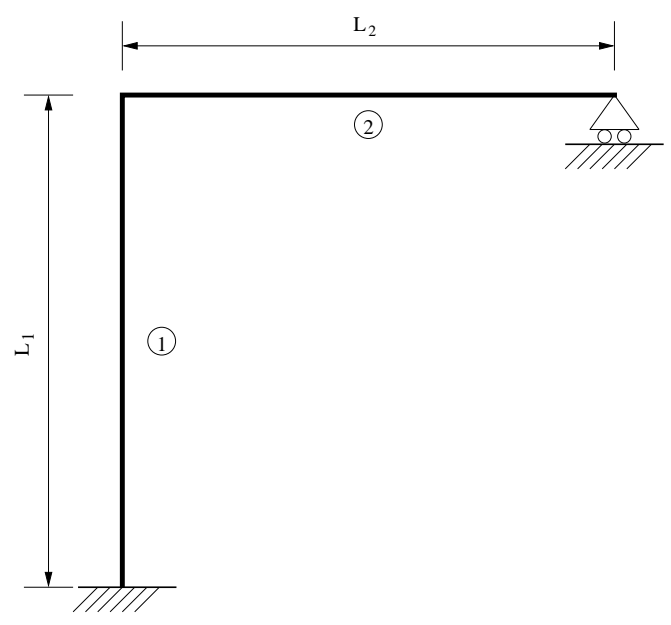

Figure 6.1: Prototype portal frame

To fix ideas, we consider the modeling of the portal frame shown in 
Fig. 6.1. All members are considered axially rigid and modeled as Timoshenko beams. The forward problem can be cast similarly to (2.2)-(2.3), stated now for each $i$ member $(i=1$ : column, $i=2$ : beam):

$$
\begin{gathered}
\frac{\partial}{\partial x_{i}}\left\{G_{i} A_{i} K_{s_{i}}\left(\frac{\partial w_{i}}{\partial x_{i}}-\psi_{i}\right)\right\}-\rho_{i} A_{i} \frac{\partial^{2} w_{i}}{\partial t^{2}}=-q_{i} \\
G_{i} A_{i} K_{s_{i}}\left(\frac{\partial w_{i}}{\partial x_{i}}-\psi_{i}\right)+\frac{\partial}{\partial x_{i}}\left(E_{i} I_{i} \frac{\partial \psi_{i}}{\partial x_{i}}\right)-\rho_{i} I_{i} \frac{\partial^{2} \psi_{i}}{\partial t^{2}}=0 .
\end{gathered}
$$

Boundary conditions:

$$
\begin{aligned}
w_{1}(0, t) & =0, \\
w_{2}(0, t) & =0, \\
w_{2}\left(L_{2}, t\right) & =0, \\
\psi_{1}(0, t) & =0, \\
G_{1} A_{1} K_{s_{1}}\left(\frac{\partial w_{1}}{\partial x}\left(L_{1}, t\right)-\psi_{1}\left(L_{1}, t\right)\right) & =0, \\
E_{2} I_{2} \frac{\partial \psi_{2}}{\partial x_{2}}\left(L_{2}, t\right) & =0,
\end{aligned}
$$

Continuity conditions:

$$
\begin{aligned}
\psi_{1}\left(L_{1}, t\right) & =\psi_{2}(0, t) \\
E_{1} I_{1} \frac{\partial \psi_{1}}{\partial x_{1}}\left(L_{1}, t\right) & =E_{2} I_{2} \frac{\partial \psi_{2}}{\partial x_{2}}(0, t),
\end{aligned}
$$

Initial conditions:

$$
\begin{aligned}
& w_{i}\left(x_{i}, 0\right)=\frac{\partial w_{i}}{\partial t}\left(x_{i}, 0\right)=0, \\
& \psi_{i}\left(x_{i}, 0\right)=\frac{\partial \psi_{i}}{\partial t}\left(x_{i}, 0\right)=0 .
\end{aligned}
$$


The frame-specific objective functional can be rewritten as:

$$
\mathcal{F}=\frac{1}{2} \sum_{i=1}^{2}\left[\sum_{j=1}^{N} \int_{0}^{T}\left(w_{i}^{m}-w_{i j}\right)^{2} \mathrm{~d} t+\mathcal{R}_{i}\left(E_{i}\right)\right]
$$

subject to (6.1)-(6.12). After side-imposing on the objective functional the Neumann-type conditions, among those in (6.3)-(6.12), the augmented Lagrangian becomes:

$$
\begin{aligned}
\mathcal{L}= & \sum_{i=1}^{2}\left[\frac{1}{2} \sum_{j=1}^{N} \int_{0}^{T}\left(w_{i}^{m}-w_{i j}\right)^{2} \mathrm{~d} t+\int_{0}^{L_{i}} \mathcal{R}_{i}\left(E_{i}\right) \mathrm{d} x_{i}\right. \\
& +\int_{0}^{T} \int_{0}^{L_{i}} \lambda_{i w}\left[\frac{\partial}{\partial x_{i}}\left\{G_{i} A_{i} K_{s_{i}}\left(\frac{\partial w_{i}}{\partial x_{i}}-\psi_{i}\right)\right\}-\rho_{i} A_{i} \frac{\partial^{2} w_{i}}{\partial t^{2}}+q_{i}\right] \mathrm{d} x_{i} \mathrm{~d} t \\
& +\int_{0}^{T} \int_{0}^{L_{i}} \lambda_{i \psi}\left[G_{i} A_{i} K_{s_{i}}\left(\frac{\partial w_{i}}{\partial x_{i}}-\psi_{i}\right)+\frac{\partial}{\partial x_{i}}\left(E_{i} I_{i} \frac{\partial \psi_{i}}{\partial x_{i}}\right)\right. \\
& \left.+\int_{0}^{L} \lambda_{i 1}\left(\dot{w}_{i}\right)_{t=0}+\lambda_{i 2}\left(\dot{\psi}_{i}\right)_{t=0} \mathrm{~d} I_{i} \frac{\partial^{2} \psi_{i}}{\partial t^{2}}\right] \mathrm{d} x_{i} \mathrm{~d} t \\
& +\int_{0}^{T} \lambda_{3}\left[G_{1} A_{1} K_{s_{1}}\left(\frac{\partial w_{1}}{\partial x}-\psi_{1}\right)\right]_{x_{1}=L_{1}}+\lambda_{4}\left[E_{2} I_{2} \frac{\partial \psi_{2}}{\partial x_{2}}\right]_{x_{2}=L_{2}} \mathrm{~d} t \\
& \left.+\int_{0}^{T} \lambda_{5}\left[E_{1} I_{1}\left(\frac{\partial \psi_{1}}{\partial x_{1}}\right)_{x_{1}=L_{1}}-E_{2} I_{2}\left(\frac{\partial \psi_{2}}{\partial x_{2}}\right)_{x_{2}=0}\right] \mathrm{d} t\right]
\end{aligned}
$$

The first-order optimality conditions for the Lagrangian (6.14) yield state, adjoint, and control problems similar to the single-member problem presented earlier. Specifically, the state and adjoint problems are each cast for the entire frame, allowing through the interface conditions the coupling of the motion of the column to the beam. The control problems, however, 
are uncoupled: each is identical to either of (3.54) or (3.55) when written for either the beam or the column members. Figures 6.2 to 6.4 present profile reconstruction results for a variety of profiles, mixing sharp and smooth profiles for the two-member frame, as well as localized damage sections for the prototype frame of Fig. 6.1. As an example of the generalization of the concepts to arbitrary-geometry frames, Fig. 6.5 depicts a two-story frame, where the response has been sampled at 4 sensors locations per member (all sensor locations are given in Table 6.1), revealing localized damage in the first-story beam, and a non-uniform modulus profile in the second-story beam, while also recovering the in-service material profile of the columns.

\begin{tabular}{|c|c|c|c|c|c|}
\hline $\begin{array}{c}\text { Sensor } \\
\text { Tag }\end{array}$ & $\mathrm{x}$ & $\mathrm{y}$ & $\begin{array}{c}\text { Sensor } \\
\text { Tag }\end{array}$ & $\mathrm{x}$ & $\mathrm{y}$ \\
\hline $\mathrm{S} 1$ & 0.0 & 0.2 & $\mathrm{~S} 2$ & 0.0 & 0.4 \\
\hline S3 & 0.0 & 0.6 & $\mathrm{~S} 4$ & 0.0 & 0.8 \\
\hline S5 & 0.0 & 0.1 & $\mathrm{~S} 6$ & 0.0 & 0.3 \\
\hline S7 & 0.0 & 0.6 & $\mathrm{~S} 8$ & 0.0 & 0.9 \\
\hline S9 & 0.1 & 2.0 & $\mathrm{~S} 10$ & 0.3 & 2.0 \\
\hline S11 & 0.5 & 2.0 & $\mathrm{~S} 12$ & 0.8 & 2.0 \\
\hline $\mathrm{S} 13$ & 1.0 & 0.1 & $\mathrm{~S} 14$ & 1.0 & 0.4 \\
\hline $\mathrm{S} 15$ & 1.0 & 0.7 & $\mathrm{~S} 16$ & 1.0 & 0.9 \\
\hline $\mathrm{S} 17$ & 1.0 & 0.2 & $\mathrm{~S} 18$ & 1.0 & 0.4 \\
\hline $\mathrm{S} 19$ & 1.0 & 0.7 & $\mathrm{~S} 20$ & 1.0 & 0.8 \\
\hline S21 & 0.1 & 1.0 & $\mathrm{~S} 22$ & 0.3 & 1.0 \\
\hline S23 & 0.5 & 1.0 & $\mathrm{~S} 24$ & 0.8 & 1.0 \\
\hline
\end{tabular}

Table 6.1: Sensor locations for the frame shown in Fig. 6.5 


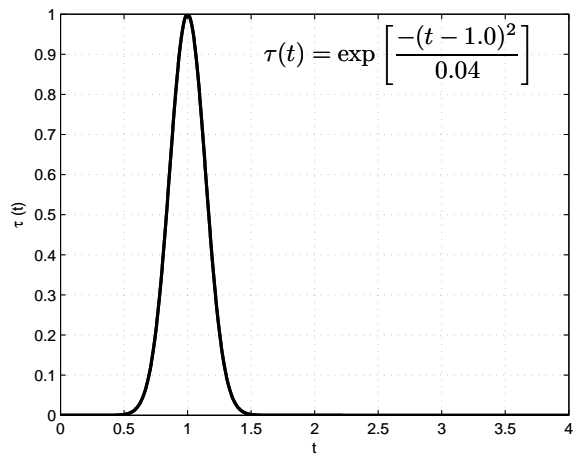

(a) Time signal $\tau(t)$

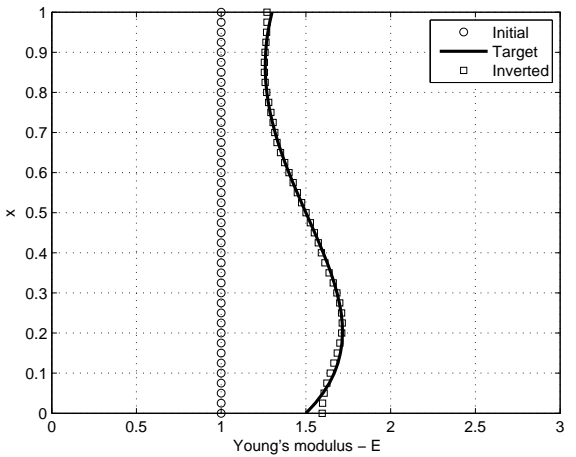

(b) Initial, target, and converged profile for the column (member 1)

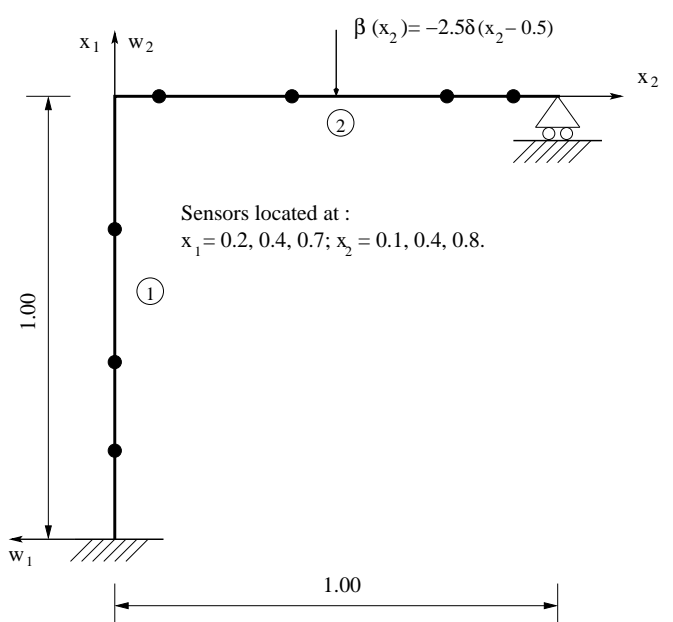

(c) Geometry, point load, and sensor locations

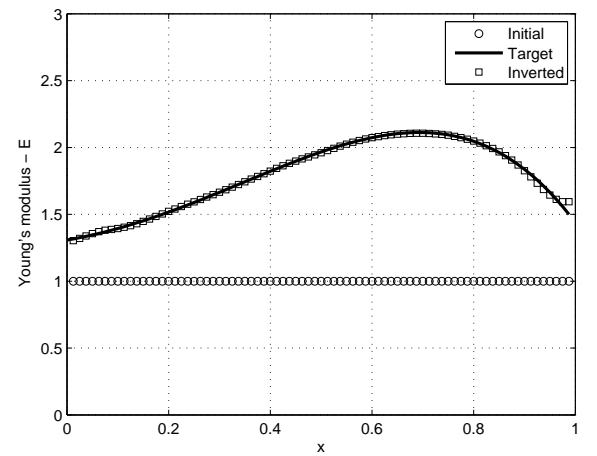

(d) Initial, target, and converged profile for the beam (member 2)

Figure 6.2: Reconstruction of a smooth target profile for a prototype portal frame: excitation, point load location, sensor distribution, TN regularization 


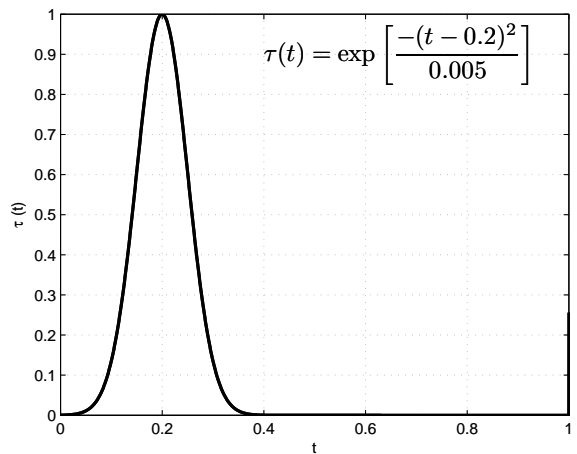

(a) Time signal $\tau(t)$

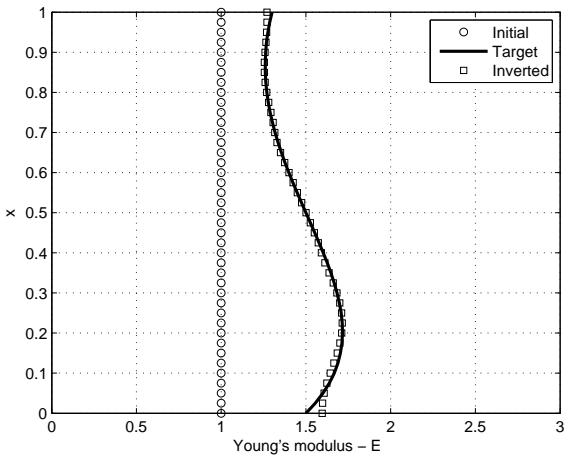

(b) Initial, target, and converged profile for the column (member 1)
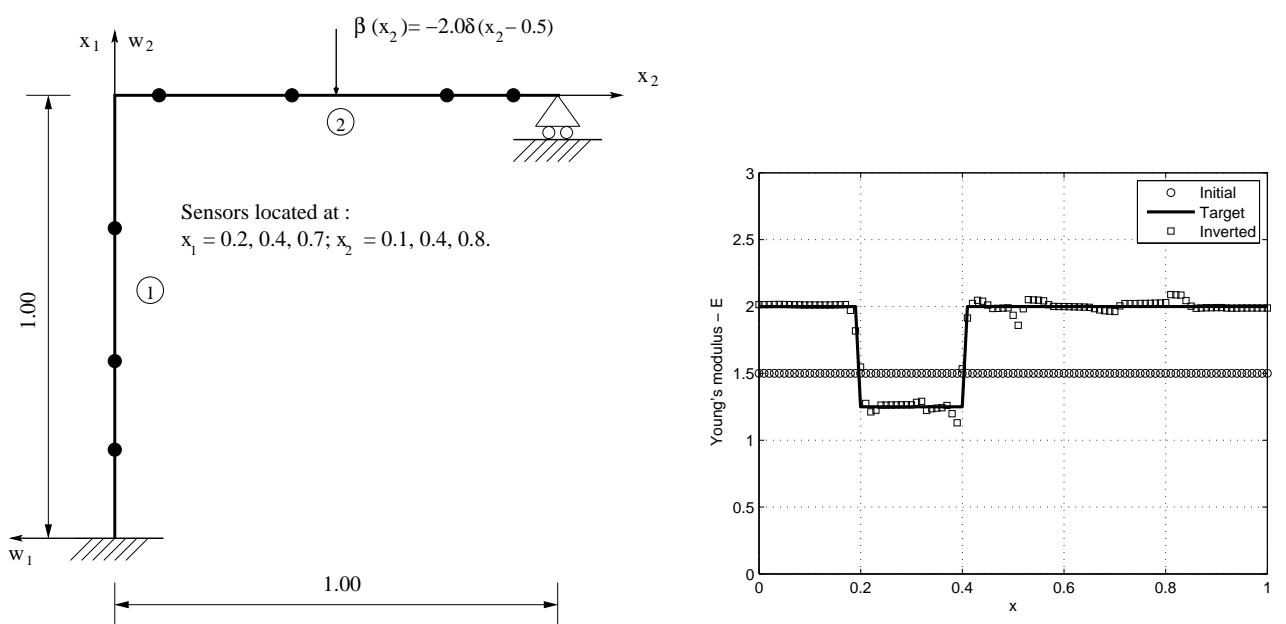

(c) Geometry, point load, and sensor loca- (d) Initial, target, and converged profile for tions the beam (member 2)

Figure 6.3: Reconstruction of a sharp target profile for a prototype portal frame: excitation, point load location, sensor distribution, TV regularization 


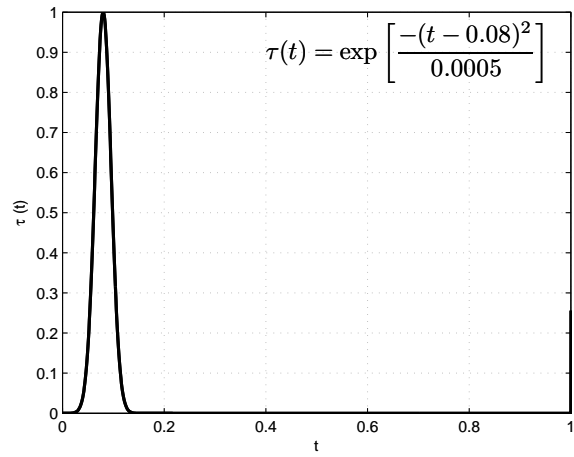

(a) Time signal $\tau(t)$

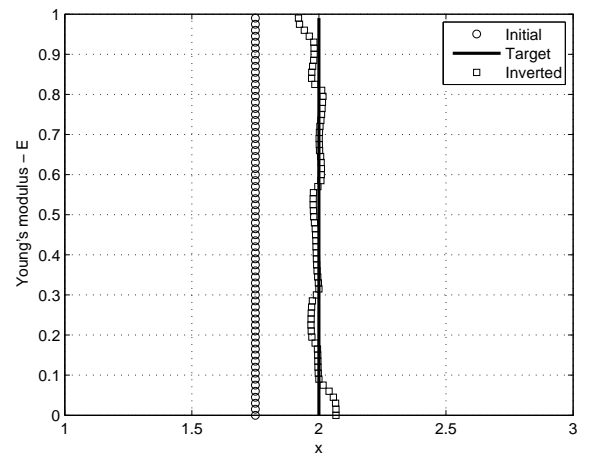

(b) Initial, target, and converged profile for the column (member 1)
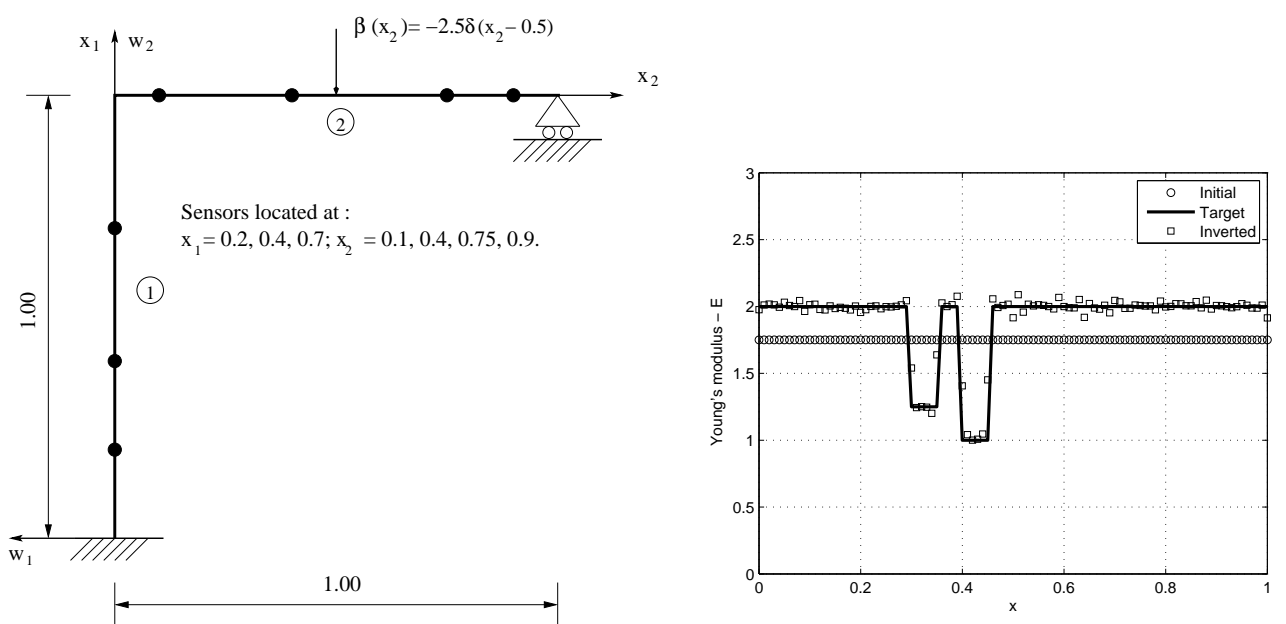

(c) Geometry, point load, and sensor loca- (d) Initial, target, and converged profile for tions the beam (member 2)

Figure 6.4: Reconstruction of localized damage for a prototype portal frame: excitation, point load location, sensor distribution, TV regularization 


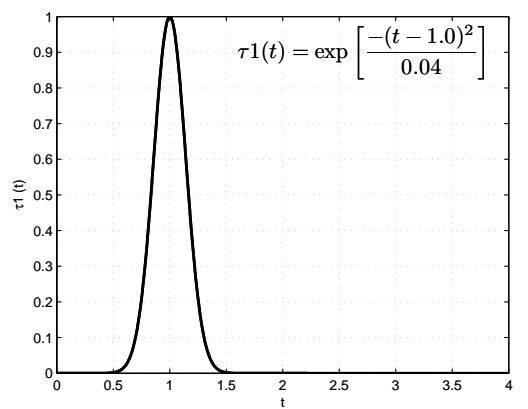

(a) Time signal $\tau_{1}(t)$ (beam B1)

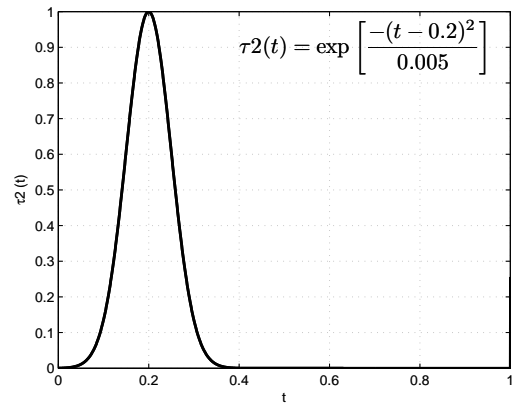

(b) Time signal $\tau_{2}(t)$ (beam B2)

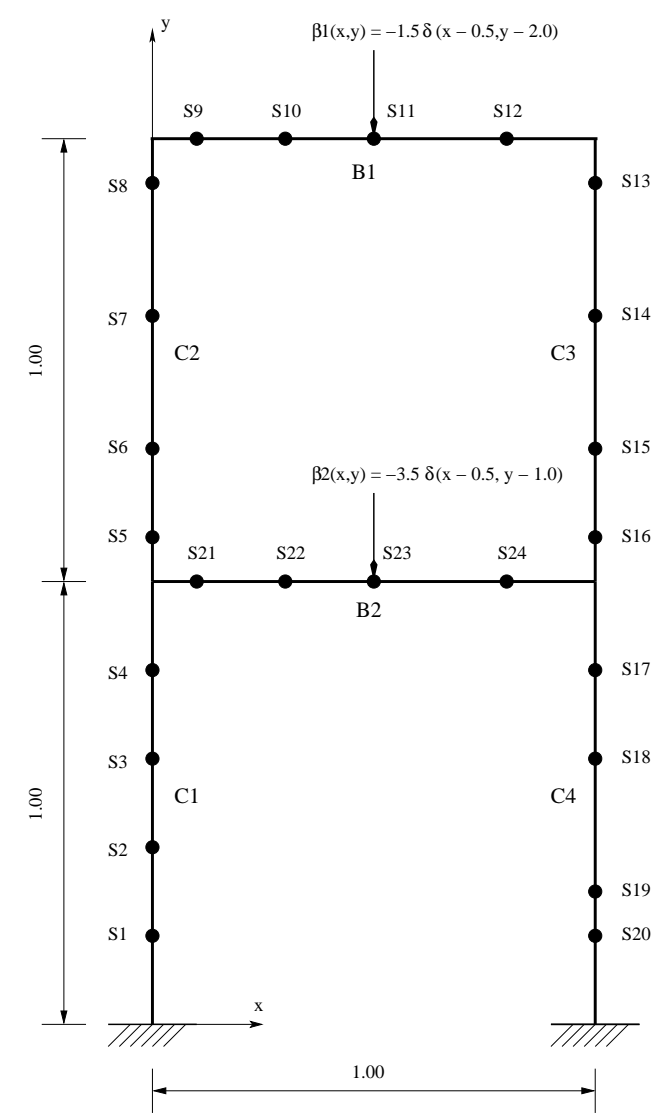

(c) Frame geometry, load, and sensor locations

Figure 6.5: Time signal, geometry, load and sensor locations for material profile reconstruction of a frame 


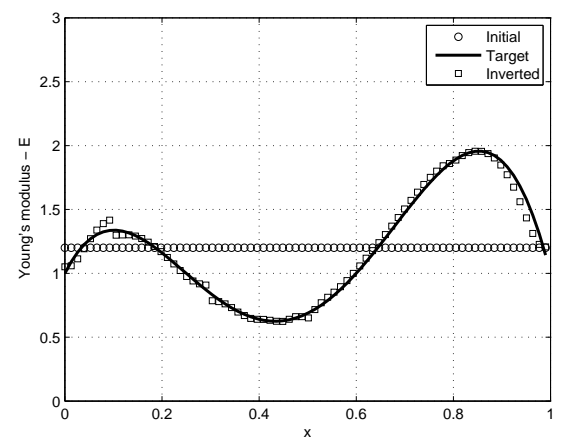

(a) Initial, target, and recovered profile (b) Initial, target, and recovered profile for beam B1

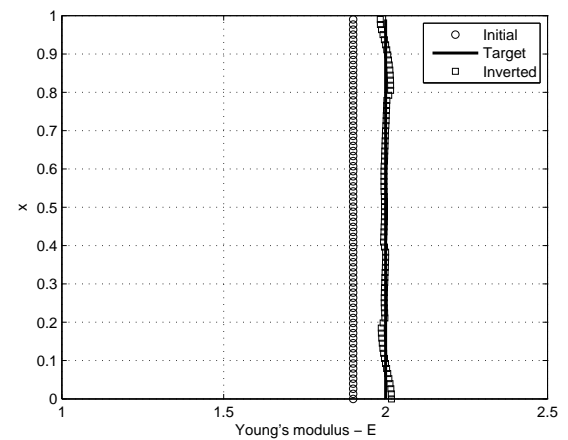

(c) Initial, target, and recovered profile (d) Initial, target, and recovered profile for column $\mathrm{C} 1$

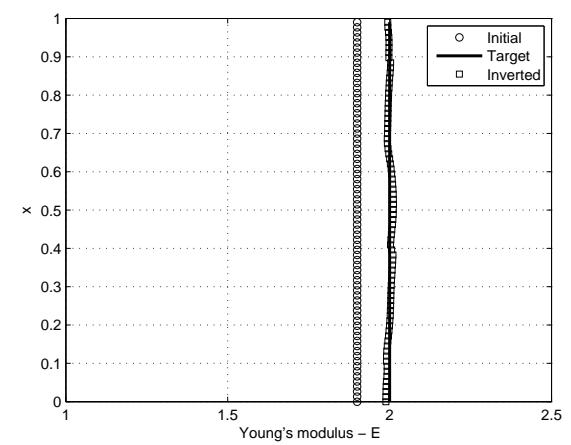

(e) Initial, target, and recovered profile (f) Initial, target, and recovered profile for column C3

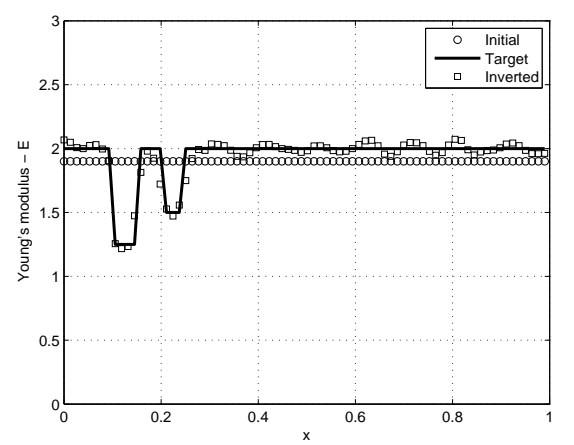
for beam B2

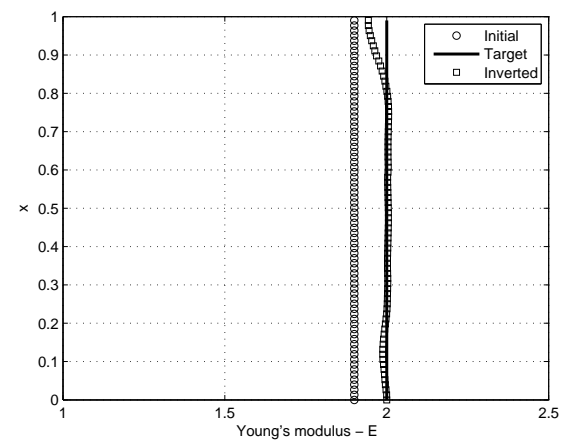

for column $\mathrm{C} 2$

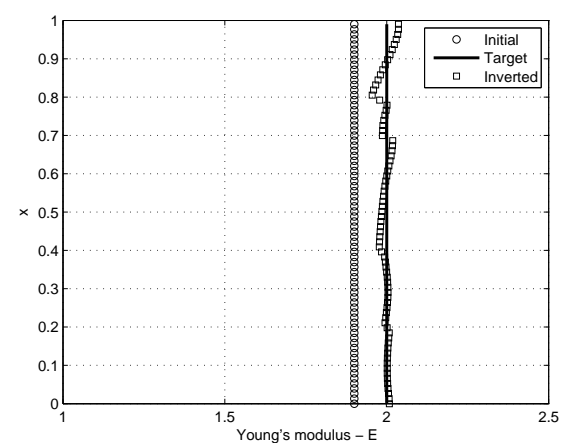
for column $\mathrm{C} 4$

Figure 6.6: Reconstruction of material profile including localized damage for the frame in Fig. 6.5 using TV regularization 


\subsection{Inversion variables other than Young's modulus}

We remark that, if instead of the Young's modulus there is interest in reconstructing the distribution of the second moment of inertia $I(x)$, as a measure of cross-sectional damage, there will be no change to the first and second optimality conditions, which will still yield the same state and adjoint problems. However, the regularization term, and the control problems will be impacted: accordingly, the control problems become:

$T N$ regularization

$$
\begin{aligned}
\delta_{I} \mathcal{L} & =-\int_{0}^{L}\left\{R_{I} \frac{d^{2} I}{d x^{2}}\right. \\
& \left.-\int_{0}^{T}\left[E \frac{\partial \psi}{\partial x} \frac{\partial \lambda_{\psi}}{\partial x}+\lambda_{\psi} \rho \frac{\partial^{2} \psi}{\partial t^{2}}\right] \mathrm{d} t\right\} \delta I \mathrm{~d} x=0
\end{aligned}
$$

subject to $\left(\frac{d I}{d x}\right)_{x=0}=0$ and $\left(\frac{d I}{d x}\right)_{x=L}=0$.

TV regularization

$$
\begin{aligned}
\delta_{I} \mathcal{L} & =-\int_{0}^{L}\left\{R_{I} \epsilon \frac{d^{2} I}{d x^{2}}\left[\left(\frac{d I}{d x}\right)^{2}+\epsilon\right]^{-\frac{3}{2}}\right. \\
& \left.+\int_{0}^{T}\left[E \frac{\partial \psi}{\partial x} \frac{\partial \lambda_{\psi}}{\partial x}+\lambda_{\psi} \rho \frac{\partial^{2} \psi}{\partial t^{2}}\right] \mathrm{d} t\right\} \delta I \mathrm{~d} x=0,
\end{aligned}
$$

subject also to $\left(\frac{d I}{d x}\right)_{x=0}=0$ and $\left(\frac{d I}{d x}\right)_{x=L}=0$.

The remainder of the inversion process for $I(x)$ is the same as the one followed for $E(x)$. 


\section{Chapter 7}

\section{Conclusions}

We presented a systematic inversion methodology for reconstructing the material profile in beams and frames of arbitrary complexity, using total wavefields for both probing and inversion purposes. We modeled the onedimensional components using Timoshenko beam theory, where both shear and rotary inertia effects are accounted for, in order to allow for a more accurate representation of the underlying wave physics when compared to EulerBernoulli assumptions.

The optimization problem was cast based on a misfit functional between the measured response and the numerically computed response. Adherence to the physics of the problem was ensured by forming an augmented Lagrangian functional, that includes the misfit, a regularization term, as well as the sideimposed governing PDEs, boundary, and initial conditions. We discussed the numerical treatment of the ensuing state, adjoint, and control problems, in the context of a reduced-space method. Moreover, Tikhonov and Total Variation regularization schemes were used to alleviate the inherent ill-posedness of the inverse medium problem.

We reported numerical results for single beams, and frames, involving 
either smooth or sharp material profiles, which in a few cases were also consistent with localized damage. Invariably, even in the presence of noise, the outlined procedure exhibited algorithmic robustness, using only a few sensors per component. We note that the process is well-suited for localizing and quantifying damage in frames without need for knowing the undamaged state or any prior state of the structure. In this context, the presented methodology offers a reasonable tool for condition assessment of existing structures. 


\section{Bibliography}

[1] R. D. Adams, P. Cawley, C. J. Pye, and B. J. Stone. A vibration technique for non-destructively assessing the integrity of structures. Journal of Mechanical Engineering Science, 20(2):93-100, 1978.

[2] B. Binici and L. F. Kallivokas. Crack identification in beams using multiple spectra. In Third European Conference on Structural Control, Vienna, Austria, 2004.

[3] T. T. Cao and D. C. Zimmerman. Application of load dependent ritz vectors for structural damage detection. In Proceedings of the 15th International Modal Analysis Conference, Orlando FL, pages 1319-1324, 1997.

[4] T. T. Cao and D. C. Zimmerman. A procedure to extract ritz vectors from dynamic testing data. In Proceedings of the 15th International Modal Analysis Conference, Orlando FL, pages 1036-1042, 1997.

[5] M. N. Cerri and F. Vestroni. Detection of damage in beams subjected to diffused cracking. Journal of Sound and Vibration, 234(2):259-276, 2000.

[6] K. F. Graff. Wave Motion in Elastic Solids. Springer Verlag, Berlin, Heidelberg, New York, 1971. 
[7] T. C. Huang. The effect of rotary inertia and of shear deformation on the frequency and normal mode equations of uniform beams with simple end conditions. Transactions of the Americal Society of Mechanical Engineers, Journal of Applied Mechanics, 28:579-584, 1961.

[8] J. W. Kang and L. F. Kallivokas. The inverse medium problem in 1D PML-truncated heterogeneous semi-infinite domains. Inverse Problem in Science and Engineering, (in review), 2009.

[9] K. K. Kapur. Vibrations of a Timoshenko beam, using finite-element approach. Journal of Acoustical Society of America, 40:1058-1063, 1966.

[10] R. Y. Liang, F. K. Choy, and J. Hu. Detection of cracks in beam structures using measurements of natural frequencies. Journal of The Franklin Institute, 328(4):505-518, 1991.

[11] J. Lions. Optimal Control of Systems Governed by Partial Differential Equations. Springer Verlag, Berlin, Heidelberg, New York, 1971.

[12] J. Nocedal and S. J. Wright. Numerical Optimization. Springer, New York, NY, 2006.

[13] A. K. Pandey, M. Biswas, and K. K. Samman. Damage detection from changes in curvature mode shapes. Journal of Sound and Vibration, 145(2):321-332, 1991. 
[14] J. N. Reddy. On locking-free shear deformable beam finite elements. Computer Methods in Applied Mechanics and Engineering, 149:113-132, 1997.

[15] P. F. Rizos, N. Aspragathos, and A. D. Dimarogonas. Identification of crack location and magnitude in a cantelever beam from the vibration modes. Journal of Sound and Vibration, 138(3):381-388, 1990.

[16] R. Ruotolo and C. Surace. Damage assessment of multiple cracked beam : numerical results and experimental validation. Journal of Sound and Vibration, 206(4):567-588, 1997.

[17] M. Sansalone and N. J. Carino. Impact echo: A method for flaw detection in concrete using transient stress waves. NBSIR, National Bureau of Standards, 86(3452):222, 1986.

[18] M. Sansalone and W. B. Streett. Impact Echo: Nondestructive Testing of Concrete and Masonry. Bullbrier Press, New Jersey Shore, PA, 1997.

[19] J. Thomas and A. H. Abbas. Finite element model for dynamic analysis of Timoshenko beam. Journal of Sound and Vibration, 41(3):291-299, 1975.

[20] A. N. Tikhonov. Solution of incorrectly formulated problems and the regularization method. Soviet Math. Doklady, 4:1035-1038, 1963. 
[21] S. P. Timoshenko. On the correction for shear of the differential equation for transverse vibrations of prismatic bars. Philosophical Magazine, 41:744-746, 1921.

[22] S. P. Timoshenko. On the transverse vibrations of bars of uniform cross section. Philosophical Magazine, 43:125-131, 1922. 


\section{Vita}

Pranav Madhav Karve, the son of Mr. Madhav K. Karve and Mrs. Vidya M. Karve, was born in Pune, India on 6 May 1983. He received Bachelor of Civil Engineering degree from the Government College of Engineering, University of Pune, India in 2005. After graduation, he joined Larsen and Toubro - Sargent and Lundy Ltd., Vadodara, India as a Graduate Engineer Trainee. He worked there, as a structural design engineer, for about two years. In the fall of 2007, he started his graduate studies in civil engineering (structures area) at the University of Texas, Austin.

Permanent address: B5 Pushkar Co-operative Hou. Society Model Colony, Pune, Maharashtra, India Pin : 411016

This thesis was typeset with $\mathrm{AT}_{\mathrm{E}} \mathrm{X}^{\dagger}$ by the author.

\footnotetext{
${ }^{\dagger} \mathrm{LATEX}$ is a document preparation system developed by Leslie Lamport as a special version of Donald Knuth's TEX Program.
} 